\title{
Multiple and Complex Codimension-2 Bifurcations underlying Post-inhibitory Rebound Spike and Excitability Transition
}

\author{
Xianjun Wang $\cdot$ Huaguang Gu四 Yuye Li $\cdot$ Bo Lu
}

Received: date / Accepted: date

\begin{abstract}
Neuron exhibits nonlinear dynamics such as excitability transition and post-inhibitory rebound (PIR) spike related to bifurcations, which are associated with information processing, locomotor modulation, or brain disease. PIR spike is evoked by inhibitory stimulation instead of excitatory stimulation, which presents a challenge to the threshold concept. In the present paper, 7 codimension- 2 or degenerate bifurcations related to 10 codimension-1 bifurcations are acquired in a neuronal model, which presents the bifurcations underlying the excitability transition and PIR spike. Type I excitability corresponds to saddle-node bifurcation on an invariant cycle (SNIC) bifurcation, and type II excitability to saddle-node (SN) bifurcation or sub-critical Hopf (SubH) bifurcation or sup-critical Hopf (SupH) bifurcation. The excitability transition from type I to II corresponds to the codimension-2 bifurcation, SaddleNode Homoclinic orbit (SNHO) bifurcation, via which SNIC bifurcation terminates and meanwhile big homoclinic orbit (BHom) bifurcation and SN bifurcation emerge. A degenerate bifurcation via which BHom bifurcation terminates and fold limit cycle (LPC) bifurcation emerges is responsible for spiking transition from type I to II, and the roles of other codimension-2 bifurcations (Cusp, Bogdanov-Takens, and Bautin) are discussed. In
\end{abstract}

Xianjun Wang, Bo Lu

School of Mathematics and Science, Henan Institute of Science and Technology, Xinxiang, 453003, China

Huaguang Gu (凶)

School of Aerospace Engineering and Applied Mechanics, Tongji University, Shanghai, 200092, China

Tel.:+86-21-65983627

E-mail: guhuaguang@tongji.edu.cn

Yuye Li

College of Mathematics and Computer Science, Chifeng University, chifeng 024000, China addition, different from the widely accepted viewpoint that PIR spike is mainly evoked near Hopf bifurcation rather than SNIC bifurcation, PIR spike is identified to be induced near SNIC or BHom or LPC bifurcations, and threshold curves resemble that of Hopf bifurcation. The complex bifurcations present comprehensive and deep understandings of excitability transition and PIR spike, which are helpful for the modulation to neural firing activities and physiological functions.

Keywords Bifurcation - Codimension-2 bifurcation . Post-inhibitory rebound spike $\cdot$ Excitability transition · Neuronal spiking

\section{Introduction}

The bifurcations have played important roles in identifying the nonlinear dynamics of neuronal electrical activities, which are involved in the physiological functions of the neural system such as information processing and locomotor control and pathology or diseases of the brain [1-9]. The neuronal electrical activities include the steady state and firing, and the transition between steady state and firing or between different firing patterns can be induced by various modulation$\mathrm{s}$ via different bifurcations [10-20]. For example, the steady state changes to firing via the sub-critical Hopf (SubH) bifurcation, or the sup-critical Hopf ( $\mathrm{SupH}$ ) bifurcation, or the saddle-node bifurcation on an invariant cycle (SNIC), or the saddle-node (SN) bifurcation $[21,22]$. Different bifurcations exhibit different dynamics when modulated by the external static stimulation [23-26]. For example, the firing frequency near SNIC point exhibits nearly zero value while near the SubH point manifests nearly fixed, nonzero value. Such different firing frequency responses have been used to in- 
terpret the type I and II excitabilities observed in the biological experiment [23]. For type I excitability, the steady state changes to firing with nearly-zero frequency, which then increases gradually with increasing stimulation strength. For type II excitability, the firing frequency emerges with nearly fixed, nonzero value. Therefore, type I and II excitabilities correspond to SNIC and SubH bifurcations related to equilibrium points, respectively. In biological experiment, type I and II excitabilities have been observed in many neurons, for example, the somatosensory neurons related to pathological pain, spinal sensory neurons, cortical pyramidal neurons, mesencephalic trigeminal neuronsgranule cells of dentate gyrus, sympathetic neurons, and hippocampal output neurons [27-37]. In addition, the type of spiking is determined by the firing frequency of spiking changed to steady state as the current strength decreases. Type I spiking exhibits nearly zero firing frequency around the disappearance of stable limit cycle, which corresponds to SNIC or big homoclinic orbit (BHom) bifurcation. Type II spiking exhibits fixed, non-zero firing frequency, which corresponds to the fold limit cycle (LPC) bifurcation or sup-critical Hopf ( $\mathrm{SupH}$ ) bifurcation. All these show that type I and II excitabilities/spikings are very important for both nonlinear dynamics and neuroscience.

Except for the firing frequency, type I and II excitabilities exhibit very different dynamics in multiple aspects $[38,39]$. For instance, the firing for type I and II excitabilities exhibit different phase response curves to the pulse stimulation [40]. Stimulated by noise, type I excitability exhibits weak coherence resonance while type II excitability manifests strong coherence resonance [26]. Network composed of neurons with type II excitability exhibits stronger synchronization than that composed of neurons with type I excitability [41, 42]. Another important example is the post-inhibitory rebound (PIR) spike evoked by the inhibitory stimulation from the steady state. The PIR spike has been widely observed in the neurons such as the neonatal rat motoneurons [43], lamprey neurons [44], interneurons in mollusk clione [45], lateral pyloric neurons of the stomatogastric ganglion [46], and superior paraolivary nucleus in the auditory brainstem [47], which is involved in information processing and locomotor modulation. It is widely accepted that the PIR spike can be evoked from the steady state near Hopf bifurcation rather than near SNIC bifurcation [22]. The PIR spike presents a challenge to the concept of the threshold. In common viewpoint, the spike or action potential is evoked by the positive or excitatory stimulation which can induce the membrane potential to increase to run across the threshold, while a negative or inhibitory s- timulation cannot evoke a spike from the steady state. The PIR spike has been observed in the biological experiments on many neurons and related to special ionic currents such as the hyperpolarization active caution current $\left(I_{h}\right)$ [48-52], which plays important roles in the physiological functions information processing and locomotor control of the neural system or brains disease such as depression [3]. In phase plane of a twodimensional neuronal model such as the famous MorrisLecar (ML) model, which is widely used to identify the dynamics of type I and II excitabilities [53-56], the threshold curve across which a spike is evoked locates down-left, below, and right to the stable focus near the Hopf bifurcation [22]. Here the phase plane is plotted by membrane potential on the horizontal axis and gating variable on the vertical axis. The down left part of the threshold curve exhibits a negative slope. When stimulated by the inhibitory pulse, the phase trajectory of the response can run across the down-left part of the threshold curve firstly and then runs to right and increases to form a PIR spike. However, such geometrical characteristics of the threshold curve vanish for the case of SNIC in the ML model (for details please refer to the Appendix and Fig. 16 of body content in the present paper). The threshold curve, which is related to the stable manifold of the saddle, mainly locates right to the stable node near the SNIC and does not exhibit negative slope. Therefore, the down-left part of the threshold curve is responsible for the generation of PIR spike for Hopf bifurcation corresponding to type II excitability. However, no PIR spike is evoked due to the absence of down-left part of the threshold curve for the SNIC with type I excitability. Recently, $I_{h}$ current induces SNIC changed to Hopf bifurcation, which further implies that the PIR spike induced by $I_{h}$ current is related to the Hopf bifurcation [57]. More recently, in a three-dimensional neuronal model with $I_{h}$ current, the PIR spike appears near SNIC because the threshold surface changes for stronger $I_{h}$ current [58]. However, up to now, no PIR spike is identified to appear near SNIC in a two-dimensional model. In addition, the PIR spike was conjectured to appear near a big homoclinic orbit (BHom) bifurcation [22]. Unfortunately, no theoretical model and examples have been provided up to now. Therefore, an important question arises. Can the PIR spike appear near SNIC in a two-dimensional neuronal or near BHom bifurcation? The answer is very important for the nonlinear dynamics and neuroscience related to the PIR spike.

Due to both types of excitability are very important, the dynamics or bifurcations related to type I excitability and type II excitability, and the dynamics or bifurcations for the transition between excitability 
types have attracted much attention in both theoretical studies [59-65] and biological experiments [27-37]. In the biological experiments, the switch between type I excitability and type II excitability was identified and induced by multiple modulations such as the M-type potassium current, A-type current, optogenetic stimulation, and in vitro or in vivo-like conditions, and so on $[28-30,35,57,58,64]$. In theoretical models, the transition between type I excitability and type II excitability has been simulated in multiple theoretical models such as the ML model, $I_{\mathrm{Na}, \mathrm{p}}+I_{\mathrm{K}}$ model [23], Leech model, and so on, and related to the autapse, $I_{h}$ current, temperature, and so on. Furthermore, the bifurcations in two-parameter space [66-70] have been used to identify dynamical mechanism of excitability transition. The codimension-2 bifurcation point, Bogdanov-Takens (BT) bifurcation, which is related to codimension-1 bifurcations such as the SN bifurcation, homoclinic orbit (Hom) bifurcation, and Hopf bifurcation, is suggested to be related to switch between type I and II excitabilities. In some Refs. [5, 62,65], the codimension-2 bifurcation point, Saddle-Node Homoclinic orbit (SNHO) bifurcation which is related to SNIC, SN, and BHom, is thought to be related to the transition between type I and II excitabilities. Therefore, the roles of codimension2 bifurcations such as BT or SNHO bifurcations in the transition between type I and II excitabilities should be further identified.

In the present paper, the codimension- 1 and -2 bifurcations underlying the PIR spike and the switch between excitability types are investigated in the $I_{\mathrm{Na}, \mathrm{p}}+$ $I_{\mathrm{K}}$ model, which is a two-dimensional neuronal model without $I_{h}$ current. Such a model has been used to study the dynamics of type I and II excitabilities [23]. Firstly, the PIR spike is identified to appear near the SNIC, BHom, and LPC bifurcations, which is related to not only the type II excitability but also the type I excitability. The PIR spike near the SNIC/type I excitability presents a novel result, and the PIR spike near the BHom bifurcation presents a demonstration of the speculation proposed in a previous study [22]. Such results present the comprehensive viewpoints of the codimension-1 bifurcations and excitability types underlying the PIR spike. Secondly, the codimension-1 bifurcations underlying the excitability types are acquired. Type I excitability corresponds to the SNIC bifurcation, and type II excitability to the SN bifurcation right to the $\mathrm{BHom}$, the $\mathrm{SubH}$ right to the $\mathrm{BHom}$, the SubH right to the LPC, and the SupH. Type I spiking corresponds to the SNIC and BHom (left to the SN and $\mathrm{SubH}$ ), and Type II spiking corresponds to the LPC (left to SubH) and SupH. Last, the codimension-2 and degenerate bifurcations underlying the PIR spike and switch between excitability types are acquired. There are multiple codimension- 1 bifurcations and 7 codimension2 or degenerate bifurcations in the $I_{\mathrm{Na}, \mathrm{p}}+I_{\mathrm{K}}$ model. Such bifurcations are more complex than those of the ML model and many other high-dimensional models such as the HH, Chay, Leech model, and so on. The excitability transition from type I to II corresponds to the codimension-2 bifurcation, Saddle-Node Homoclinic orbit (SNHO) bifurcation, via which the SNIC terminates, and meanwhile the BHom and SN bifurcations emerge. A degenerate bifurcation same as $\mathrm{P}_{1}$ in Ref. [54], via which the BHom terminates and LPC emerges, is responsible for the spiking transition from type I to II. The roles of other codimension-2 bifurcations such as the Cusp bifurcation (CP), Bogdanov-Takens bifurcation (BT), and Bautin bifurcation (GH) are discussed. The novel bifurcation and excitability mechanisms for the PIR phenomenon and the comprehensive bifurcation mechanisms for the excitability transition are helpful for the modulations to the neural firing activities and physiological functions.

The rest of the present paper are organized as follows. Section 2 presents the model and method. The results are provided in Section 3. Section 4 presents the conclusion and discussion.

\section{Model and Method}

\subsection{Model}

The $I_{\mathrm{Na}, \mathrm{p}}+I_{\mathrm{K}}$ model [23] has been used to investigate the dynamics of excitability, which consists of a persistent $\mathrm{Na}^{+}$current, a relatively slower persistent $\mathrm{K}^{+}$ current, and a leak current, reading as follows:

$$
\text { (1) }\left\{\begin{aligned}
C \frac{d V}{d t} & =I+I_{\mathrm{Na}}+I_{\mathrm{K}}+I_{\mathrm{L}}, \\
\frac{d n}{d t} & =\frac{n_{\infty}(V)-n}{\gamma(V)},
\end{aligned}\right.
$$

where $V$ is the membrane potential with unit $\mathrm{mV}$ and $n$ denotes the unitless gating variable for $\mathrm{K}^{+}$channel. The parameter $C$ represents the capacitance of a neuron and $I$ denotes a constant current. $I_{\mathrm{Na}}=-g_{\mathrm{Na}} m_{\infty}(V)(V-$ $\left.E_{\mathrm{Na}}\right), I_{\mathrm{K}}=-g_{\mathrm{K}} n\left(V-E_{\mathrm{K}}\right)$ and $I_{\mathrm{L}}=-g_{\mathrm{L}}\left(V-E_{\mathrm{L}}\right)$ denote the ionic currents of $\mathrm{Na}^{+}, \mathrm{K}^{+}$, and leakage ion respectively. $E_{\mathrm{Na}}, E_{\mathrm{K}}, E_{\mathrm{L}}$ are the corresponding reversal potential and $g_{\mathrm{Na}}, g_{\mathrm{K}}, g_{\mathrm{L}}$ are the corresponding maximal conductances. The functions $m_{\infty}$ and $n_{\infty}$ are steady state of the gating variables of $\mathrm{Na}^{+}$and $\mathrm{K}^{+}$, respectively, and $\gamma$ is the time factor, where $m_{\infty}(V)=$ $\frac{1}{1+\exp \left(\left(V_{1 / 2 \_m}-V\right) / k_{m}\right)}, n_{\infty}(V)=\frac{1}{1+\exp \left(\left(V_{1 / 2 \_n}-V\right) / k_{n}\right)}$, and $\gamma(V)=1$. 
In the present paper, $I$ is the bifurcation parameter and $V_{1 / 2 \_n}$ the control parameter, and the other parameter values are $k_{n}=7 \mathrm{mV}, V_{1 / 2 \_m}=-20 \mathrm{mV}, k_{m}=15$ $\mathrm{mV}, C=1 \mu \mathrm{F} / \mathrm{cm}^{2}, E_{\mathrm{L}}=-79.42 \mathrm{mV}, E_{\mathrm{K}}=-90$ $\mathrm{mV}, E_{\mathrm{Na}}=60 \mathrm{mV}, g_{\mathrm{L}}=8 \mathrm{mS} / \mathrm{cm}^{2}, g_{\mathrm{K}}=10 \mathrm{mS} / \mathrm{cm}^{2}$, $g_{\mathrm{Na}}=20 \mathrm{mS} / \mathrm{cm}^{2}$. And the unit of current is $\mu \mathrm{A} / \mathrm{cm}^{2}$.

\subsection{Threshold curve and the PIR spike}

The threshold is an important characteristic of the neuronal electrical activity especially for the steady state. In common viewpoint, when an excitatory or depolarization stimulation is applied to a steady state, the membrane potential increases to go beyond a threshold to form an action potential. If the stimulation is weak, the membrane potential increases, but fails to reach the threshold, thus no action potential but subthreshold membrane potential appears. This is the common viewpoint of the voltage threshold.

In the present paper, the voltage threshold for the action potential evoked from the steady state is studied. For the $I_{\mathrm{Na}, \mathrm{p}}+I_{\mathrm{K}}$ model, if an initial value $(V, n)$ for the Eq.(1) induces an action potential, such an initial value is called suprathreshold and labeled as blank in the phase plane. The initial value $(V, n)$, which cannot induce an action potential, is called subthreshold and labeled with yellow in the phase plane. The border between the yellow and blank areas in the phase plane is the threshold curve. In the present paper, if the maximal value of the membrane potential is greater than 0 $\mathrm{mV}$, an action potential is thought to appear. Obviously, the phase point of the steady state locates within the yellow area. The phase trajectory from the steady state to an action potential, which is evoked from the steady state, runs across the threshold curve, because a part of the action potential such as the peak locates within the blank area. And the phase point of the steady state and the subthreshold membrane potentials evoked from the steady state locate within the yellow area. Therefore, the threshold curve is very important to identify the generation of an action potential evoked from the steady state, especially for the PIR spike.

Except for the well-known excitatory stimulation, if an inhibitory stimulation can elicit an action potential or spike from the steady state, i.e. the trajectory of the electrical behavior following the stimulation run across the threshold curve, then the PIR spike is evoked. In the present paper, the relationship between the threshold curve and PIR spike is studied. It should be emphasized here that the phase plane is always plotted with the membrane potential $V$ horizontally and gating variable $n$ vertically, which is aimed to keep consistent with that in Ref. [22] and thus is convenient to make a comparison.

\subsection{The inhibitory stimulation}

In the present paper, we adopt inhibitory (i.e., negative) pulse stimulation, denoted by $I_{\text {stim }}$, to the steady state to study the PIR spike. Adding $I_{\text {stim }}$ into the right hand of the first equation in Eq. (1), one has

$$
\text { (2) }\left\{\begin{aligned}
C \frac{d V}{d t} & =I+I_{\mathrm{Na}}+I_{\mathrm{K}}+I_{\mathrm{L}}+I_{\text {stim }}, \\
\frac{d n}{d t} & =\frac{n_{\infty}(V)-n}{\gamma(V)}
\end{aligned}\right.
$$

When initial value $(V, n)$ is set to be the value of the steady state, Eq. (2) governs the response to the inhibitory pulse stimulation. Throughout the whole paper, $I_{\text {stim }}$ is a simple pulse with application time at $50 \mathrm{~ms}$ and termination time at $51.3 \mathrm{~ms}$, i.e., duration fixed at $1.3 \mathrm{~ms}$. And the pulse strength is labeled with $A(<0) \mu \mathrm{A} / \mathrm{cm}^{2}$.

\subsection{Method}

Fourth-order Runge-Kutta method is utilized to integrate the equations of the model with time step 0.001 ms. The bifurcation analysis is applied to Eq. (1) and performed by software XPPAUT [71], including oneparameter and two-parameter bifurcations.

\section{Results}

\subsection{The electrical behaviors}

The $I_{\mathrm{Na}, \mathrm{p}}+I_{\mathrm{K}}$ model exhibits three different types of behaviors, i.e., the resting state, the period-1 firing, and the depolarization block. For example, at $V_{1 / 2 \_n}=-29$ $\mathrm{mV}$, the representatives for the 3 behaviors are depicted in Fig. 1(a) $\left(I=3 \mu \mathrm{A} / \mathrm{cm}^{2}\right.$, stable node corresponding to resting state), Fig. 1 (b) $\left(I=100 \mu \mathrm{A} / \mathrm{cm}^{2}\right.$, stable limit cycle corresponding to period-1 firing), and Fig. 1(c) ( $I=240 \mu \mathrm{A} / \mathrm{cm}^{2}$, stable focus corresponding to depolarization) respectively. The membrane potential of the resting state and depolarization block are about -59.83 $\mathrm{mV}$ (Fig. 1(a)) and about $-18.98 \mathrm{mV}$ (Fig. 1(c)). In the present paper, the dynamics near the resting state and the period-1 firing and transition between them are studied. 

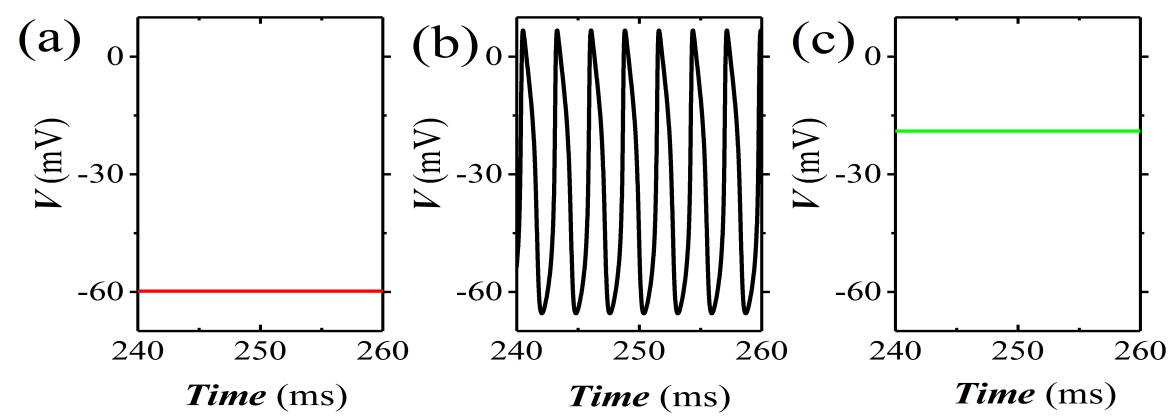

Fig. 1: Three dynamical behaviors of $I_{\mathrm{Na}, \mathrm{p}}+I_{\mathrm{K}}$ model at $V_{1 / 2 \_n}=-29 \mathrm{mV}$. (a) Resting state with $I=3 \mu \mathrm{A} / \mathrm{cm}^{2}$; (b) Period-1 firing with $I=100 \mu \mathrm{A} / \mathrm{cm}^{2}$; (c) Depolarization block with $I=240 \mu \mathrm{A} / \mathrm{cm}^{2}$.
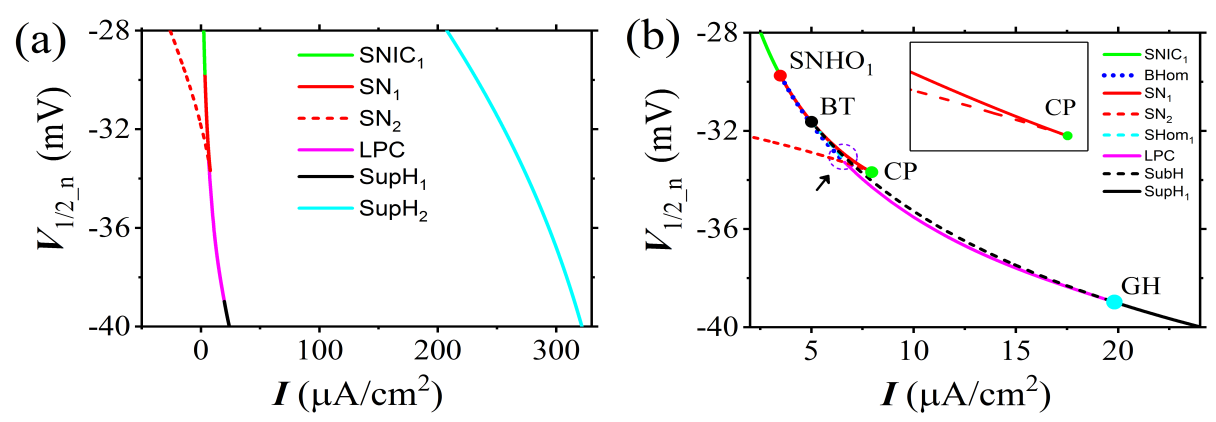

Fig. 2: Two-parameter bifurcation diagram in the $\left(I, V_{1 / 2 \_n}\right)$ plane. (a) The global view. (b) The enlargement of (a) for the left part. The $\mathrm{SNHO}_{1}$ (red solid circle) denotes the Saddle-Node Homoclinic orbit bifurcation and GH (cyan solid circle) for Bautin bifurcation. BT (black solid circle) is short for Bogdanov-Takens bifurcation, and CP (green solid circle) for Cusp bifurcation. The $\mathrm{SNIC}_{1}$ (green solid line) represents the curve of saddle-node bifurcation on an invariant circle. SubH (black dashed line) denotes sub-critical Hopf bifurcation curve. $\mathrm{SupH}_{2}$ (cyan solid curve) and $\mathrm{SupH}_{1}$ (black solid curve) represents two different curves of sup-critical Hopf bifurcation. BHom (blue dotted line) and $\mathrm{SHom}_{1}$ (cyan dashed line) represent the saddle homoclinic orbit bifurcation curves. $\mathrm{SN}_{1}$ (red solid line) and $\mathrm{SN}_{2}$ (red dashed line) are curves of the saddle-node bifurcation of equilibria. LPC (magenta solid line) represents the curve of fold limit cycle bifurcation. The insert shows details around the CP. Two degenerate bifurcation points and another Saddle-Node Homoclinic orbit bifurcation point locate in the big dashed circle (at black arrow).

\subsection{Two-parameter bifurcation diagram}

To acquire comprehensive view of bifurcation phenomena, bifurcations in the plane $\left(I, V_{1 / 2 \_n}\right)$ are acquired, as shown in Fig. 2. A groups of codimension-1 bifurcation curves locates in the left part and a sup-critical Hopf ( $\mathrm{SupH}_{2}$, cyan) bifurcation locates at higher $I$ value. The $\mathrm{SupH}_{2}$ is related to the bifurcation from limit cycle to depolarization block, which is not the focus of the present paper. The codimension-1 bifurcation curves in left part are enlarged in Fig. 2(b), which is the focus of the present paper.

Fig. 3(a), (b), (c), and (d) represent local enlargement of Fig. 2(b) around different codimension-2 or degenerate bifurcation points, where the gray region is for the mono-stable equilibrium, pink region for the stable limit cycle, blank region for the coexistence of stable equilibrium and stable limit cycle. There are 10 codimension-1 bifurcation curves, including $\mathrm{SNIC}_{1}$, BHom, $\mathrm{SN}_{1}, \mathrm{SN}_{2}, \mathrm{SHom}_{1}, \mathrm{LPC}, \mathrm{SubH}, \mathrm{SupH}_{1}, \mathrm{SNIC}_{2}$, and $\mathrm{SHom}_{2}$, respectively. Here $\mathrm{SNIC}_{1}$ and $\mathrm{SNIC}_{2}$ are for saddle-node bifurcation on an invariant circle, LPC for fold bifurcation of limit cycle, SubH for sub-critical Hopf, $\mathrm{SupH}_{1}$ for sup-critical Hopf, $\mathrm{SN}_{1}$ and $\mathrm{SN}_{2}$ for saddle-node bifurcation, BHom and $\mathrm{SHom}_{1}$ and $\mathrm{SHom}_{2}$ for homoclinic bifurcation. There are 7 codimension2 or degenerate bifurcation points, which are intersection points of some codimension-1 bifurcation curves. Four out of them are the well-known Saddle-Node Homoclinic orbit bifurcation $\left(\mathrm{SNHO}_{1}\right.$, red solid circle), Bogdanov-Takens bifurcation (BT, black solid circle), Cusp bifurcation (CP, red solid circle), and Bautin bifurcation (GH, cyan solid circle), as shown in Fig. 2(b). Other three points locate within the big dashed circle (at the black arrow) shown in Fig. 2(b), which are two degenerate bifurcation points $\mathrm{P}_{1}$ (Fig. $3(\mathrm{c})$ ) and $\mathrm{N}$ 

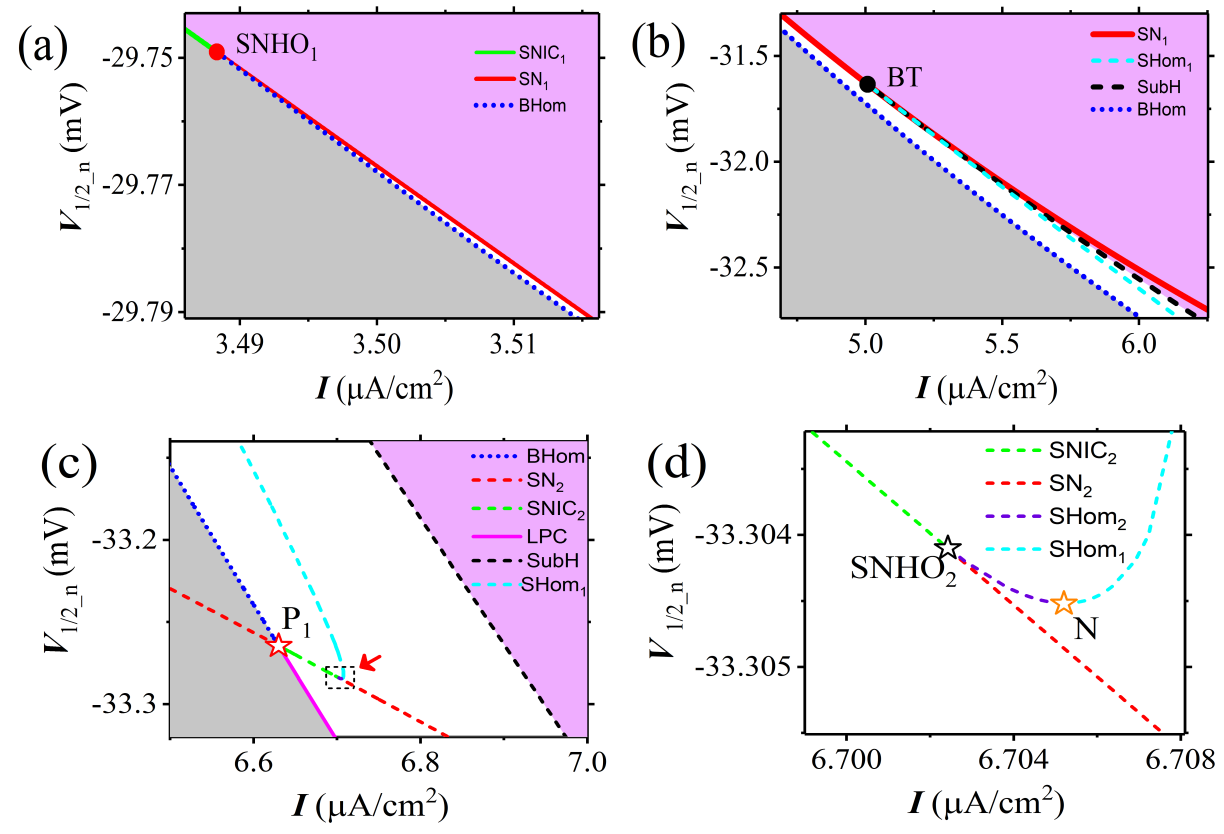

Fig. 3: The two-parameter bifurcation diagrams around different codimension-2 or degenerate bifurcation points. (a) Around $\mathrm{SNHO}_{1}$ point of Fig. 2(b); (b) Around BT point of Fig. 2(b); (c) In the big dashed circle (at black arrow) of Fig. 2(b). The degenerate bifurcation point $\mathrm{P}_{1}$ (red hollow star) is responsible for the change from BHom to LPC bifurcation; (d) In the dashed rectangle (at red arrow) of Fig. 3(c). The curve SHom 2 (purple dashed) connects points $\mathrm{SNHO}_{2}$ (black hollow star) and a degenerate bifurcation point $\mathrm{N}$ (orange hollow star). The gray, pink, and blank region denotes the mono-stable equilibrium, stable limit cycle, and the coexistence.

(Fig. 3(d)), and a Saddle-Node Homoclinic orbit bifurcation $\mathrm{SNHO}_{2}$ (Fig. 3(d)). The bifurcation phenomena around these 7 points are introduced in the following paragraphs.

Codimension-2 bifurcation point $\mathbf{S N H O}_{1}$ Shown in Fig. 3(a) is the enlargement of Fig. 2(b) around the point $\mathrm{SNHO}_{1}$ (red solid circle), which correspond$\mathrm{s}$ to the intersection of the codimension-1 bifurcation curves $\mathrm{SN}_{1}$ (red solid line), BHom (blue dotted line), and $\mathrm{SNIC}_{1}$ (green solid line). Via $V_{1 / 2 \_n}$ decreasing across $\mathrm{SNHO}_{1}$, the curve $\mathrm{SNIC}_{1}$ (green solid line) changes to curve $\mathrm{SN}_{1}$, and meanwhile the curve BHom emerges. Upper to the point $\mathrm{SNHO}_{1}$, the curve $\mathrm{SNIC}_{1}$ separates exactly the steady state (gray) and spiking (pink) and there is no coexistence behavior. Lower to the $\mathrm{SNHO}_{1}$, the coexistence behavior (blank region) appears and locates between curves $\mathrm{BHom}$ and $\mathrm{SN}_{1}$. Therefore, $\mathrm{SNHO}_{1}$ corresponds to the appearance of the BHom bifurcation and the coexistence behavior with decreasing $V_{1 / 2 \_n}$.

Codimension-2 bifurcation point BT As $V_{1 / 2 \_n}$ further decreases, the $\mathrm{SN}_{1}$ curve related to the $\mathrm{SNHO}_{1}$ bifurcation point (Fig. 3(a)) runs across the BT point and then the SubH curve (black dashed line) and $\mathrm{SHom}_{1}$ curve (cyan dashed line) emerge from the BT point. Therefore, the BT bifurcation is responsible for the e- mergence of the SubH (black dashed line)and the $\mathrm{SHom}_{1}$ (cyan dashed line) bifurcations, as shown in Fig. 3(b). The coexistence behavior locates between curves BHom and $\mathrm{SN}_{1}$ upper to BT, whereas between curves BHom and SubH lower to BT. That is to say, the left boundary of the coexistence behavior (blank region) corresponds to curve BHom and does not change, whereas the right boundary changes from curve $\mathrm{SN}_{1}$ to curve $\mathrm{SubH}$.

Degenerate bifurcation point $\boldsymbol{P}_{1}$ Fig. 3(c) shows the details in the big dashed circle depicted in Fig. 2(b). With decreasing $V_{1 / 2 \_n}$, the curve BHom (blue dotted) terminates and the curve LPC (magenta solid) appears via point $\mathrm{P}_{1}$ (red star). Meanwhile, the curve $\mathrm{SN}_{2}$ (dashed red) terminates and a saddle-node bifurcation on an invariant cycle (labeled as $\mathrm{SNIC}_{2}$, green dashed) curve appears through the $\mathrm{P}_{1}$ point. Such a point $\mathrm{P}_{1}$ has been reported in Morris-Lecar model (Fig. 6 of [54]), which is thought to be a degenerate bifurcation point therein. Moreover, one can find that the left boundary of the coexistence behavior (blank region) changes from curve BHom to curve $\mathrm{LPC}$ via point $\mathrm{P}_{1}$, whereas the right boundary is still curve SubH.

Codimension-2 bifurcation point $\mathrm{SNHO}_{2}$ and degenerate bifurcation point $\boldsymbol{N}$ Shown in Fig. 3(d) are the details of Fig. 3(c) within the dashed rectangle 
(corresponding to red arrow). With decreasing $V_{1 / 2 \_n}$, the $\mathrm{SNIC}_{2}$ curve (green dashed line) changes to the $\mathrm{SN}_{2}$ curve (red dashed) via the point $\mathrm{SNHO}_{2}$ (black hollow star), meanwhile a $\mathrm{SHom}_{2}$ curve (purple dashed line) appears. The curve $\mathrm{SHom}_{2}$ (purple dashed) emerging from the $\mathrm{SNHO}_{2}$ bifurcation and the $\mathrm{SHom}_{1}$ curve (cyan dashed line) emerging from the point BT (Fig. $3(\mathrm{~b})$ ) contact with each other to form a degenerate bifurcation, labeled with $\mathrm{N}$ (orange hollow star) in the present paper, as shown in Fig. 3(d). Such a point N corresponds to the disappearance (or appearance) of curves $\mathrm{SHom}_{2}$ and $\mathrm{SHom}_{1}$. The dynamics around the $\mathrm{SNHO}_{2}$ point and $\mathrm{N}$ point resemble those around the degenerate bifurcation point $\mathrm{P}_{2}$ in Fig. 6 of Ref [54].

Codimension-2 bifurcation point $\boldsymbol{G H}$ With decreasing $V_{1 / 2 \_n}$, the SubH curve (black dashed line) emerging from the BT point (black solid circle, Fig. 2(b) and Fig. 3(b)) and the LPC curve (magenta solid line) emerging from the degenerate bifurcation point $\mathrm{P}_{1}$ (red star, Fig. 3(c)) contact with each other at point $\mathrm{GH}$ (cyan solid circle) and then disappear, and meanwhile a sup-critical Hopf bifurcation curve $\left(\mathrm{SupH}_{1}\right.$, black solid curve) appears, as shown in Fig. 2(b). The coexistence behavior locates between curves LPC (magenta solid) and SubH (black dashed) when $V_{1 / 2 \_n}$ is upper to GH, whereas disappears when $V_{1 / 2 \_n}$ is lower to GH. That is to say, $\mathrm{GH}$ is responsible for the disappearance of the coexistence behavior.

Codimension-2 bifurcation point $\boldsymbol{C P}$ The Cusp bifurcation $(\mathrm{CP})$ is the intersection of codimension-1 bifurcation curves $\mathrm{SN}_{1}$ and $\mathrm{SN}_{2}$, as shown in Fig. 2(b) and the insert figure therein, which corresponds to the disappearance of both $\mathrm{SN}_{1}$ and $\mathrm{SN}_{2}$ with decreasing $V_{1 / 2 \_n}$.

These bifurcation phenomena can be further well interpreted by one-parameter bifurcation diagram with respect to $I$ at different $V_{1 / 2 \_n}$ values, which are introduced in following subsection.

3.3 One-parameter bifurcation diagram and types of (spiking, excitability)

In this subsection, we explain the bifurcation dynamics around above 7 points in two-parameter bifurcation plane by showing one-parameter bifurcation diagrams with respect to $I$. Meanwhile, the corresponding change of firing frequency is acquired to describe the spiking type and excitability type. In the present paper, to show the detailed excitability type as current $I$ is increased and spiking type as current $I$ is decreased, the notation (A, B) is adopted, where A is either I or II for spiking type, and B is either I or II for excitability type. The spiking type and excitability type here are consistent with those of Refs. [22,23]. Such a notation (A,B) can be conveniently used to describe the types of (spiking, excitability). For example, (I, II) means type I spiking and type II excitability.

\subsubsection{One-parameter bifurcation diagrams around $\mathrm{SNHO}_{1}$ : the excitability type transition}

When $V_{1 / 2 \_n}=-29$ (upper to $\mathrm{SNHO}_{1}$ point), the SNIC bifurcation with respect to $I$ appears, as shown in Fig. 4(a1), and Fig. 4(a2) and (a3) represent the details of Fig. 4(a1) around $\mathrm{SNIC}_{1}$ point (green solid circle). The equilibrium is depicted by the blue $S$-shaped curve, where the upper branch (short for UB) represents the unstable focus (long dashed line) and stable focus (solid blue line) separated by the sup-critical Hopf bifurcation point $\mathrm{SupH}_{2}$ (black solid circle), the middle branch (MB) denotes the saddle (dotted line), and the lower branch (LB) is formed by the stable focus (thick blue) and stable node (thin blue). The extreme values of stable limit cycle bifurcated from the $\mathrm{SupH}_{2}$ are denoted by the lower and upper red curves. The limit cycle contacts with the intersection point (green solid circle) of the $\mathrm{MB}$ and $\mathrm{LB}$ at $I \approx 3.03631 \mu \mathrm{A} / \mathrm{cm}^{2}$ to form the $\mathrm{SNIC}_{1}$ bifurcation (indicated by the vertical dashed line), which corresponds to the codimension-1 bifurcation curve $\mathrm{SNIC}_{1}$ (green) in Fig. 2 and Fig. 3(a). The intersection of the UB and MB is for a saddle-node (SN) bifurcation point, denoted by $\mathrm{SN}_{2}$ (red hollow circle), which corresponds to the codimension-1 bifurcation curve $\mathrm{SN}_{2}$ (red dashed) in Fig. 2. The symbol FN (at red arrow) on the LB denotes the transition between the stable focus and stable node. The stable equilibrium on LB and the stable limit cycle correspond to the gray area and pink area in Fig. 3(a) respectively. No coexistence of the firing and stable node appears near the SNIC, which is consistent with the result shown in Fig. 2 or Fig. 3(a). Here the bifurcation point $\mathrm{SupH}_{2}$ (black solid circle) on UB corresponds to the codimension-1 bifurcation curve $\mathrm{SupH}_{2}$ (cyan) in Fig. 2(a).

When $V_{1 / 2 \_n}=-29.8$, which is lower to $\mathrm{SNHO}_{1}$ point, the big homoclinic orbit (BHom) bifurcation emerges, labeled as BHom (at the black arrow) in Fig. 4(b1-b3), where Fig. 4(b2) and (b3) represents the details of Fig. 4(b1) around the red solid circle (labeled as $\left.\mathrm{SN}_{1}\right)$. The BHom bifurcation occurs at $I=I_{\mathrm{BHom}}$ $\left(\approx 3.5204736 \mu \mathrm{A} / \mathrm{cm}^{2}\right)$, as indicated by the vertical dashed line with arrow, and is formed by the intersection between the limit cycle (red) and saddle on the M$\mathrm{B}$, and meanwhile the stable node on LB is within such a homoclinic loop (more details are shown in Fig. 5). The symbol $\mathrm{SN}_{1}$ is the intersection between the LB and MB of the $S$-shaped curve, and denotes the saddle-node 

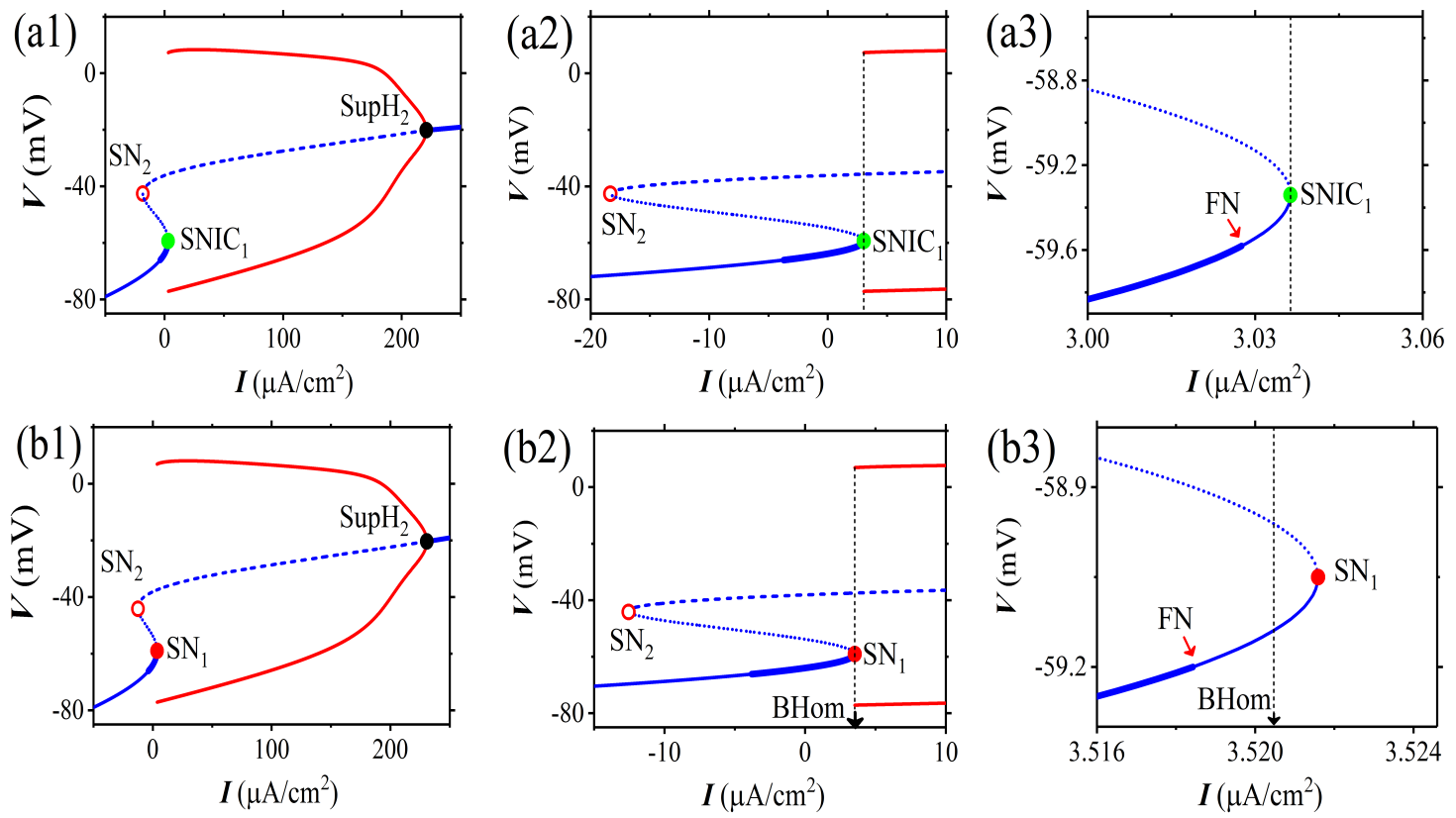

Fig. 4: The one-parameter bifurcation with respect to $I$ at different $V_{1 / 2 \_n}$ values around the codimension-2 bifurcation point $\mathrm{SNHO}_{1}$ in Fig. 2. The equilibrium is depicted by the blue $S$-shaped curve, where the upper branch (short for UB) represents the unstable equilibrium (long dashed line) and stable focus (thick blue), the middle branch (MB) denotes the saddle (dotted line), and the lower branch (LB) is formed by the stable focus (thick blue) and stable node (thin blue). The intersection of the UB and MB is for a saddle-node (SN) bifurcation point, denoted by $\mathrm{SN}_{2}$ (red hollow circle) in the present paper. The symbol FN (at red arrow) on the LB denotes the transition between the stable focus and stable node. The upper and lower red solid lines are for the extreme values of the stable limit cycle, which emerges from the sup-critical Hopf ( $\mathrm{SupH}_{2}$, black solid circle ) bifurcation. (a1) and its enlargements (a2-a3) correspond to $V_{1 / 2 \_n}=-29 \mathrm{mV}$. The green solid circle, i.e., the intersection of the $\mathrm{MB}$ and $\mathrm{LB}$ at $I \approx 3.03631 \mu \mathrm{A} / \mathrm{cm}^{2}$ and meanwhile the emergence of a limit cycle form the SNIC bifurcation (labeled as $\mathrm{SNIC}_{1}$ ), as indicated by the vertical dashed line. (b1) and its enlargements (b2-b3) correspond to $V_{1 / 2 \_n}=-29.8 \mathrm{mV}$. The limit cycle (red solid line) appears via the BHom bifurcation (dashed vertical line) at $I_{\mathrm{BHom}} \approx 3.5204736 \mu \mathrm{A} / \mathrm{cm}^{2}$, and the intersection between the $\mathrm{LB}$ and $\mathrm{MB}$ forms a saddle-node $\left(\mathrm{SN}_{1}\right.$, red solid circle) bifurcation.

bifurcation and occurs at $I \approx 3.52159 \mu \mathrm{A} / \mathrm{cm}^{2}$. Here the bifurcation points BHom and $\mathrm{SN}_{1}$ correspond to the codimension-1 bifurcation curves BHom (blue dotted) and $\mathrm{SN}_{1}$ (red solid) in Fig. 2 or Fig. 3(a). One finds clearly that the BHom appears left to the $\mathrm{SN}_{1}$, and thus the coexistence of the firing and stable node appears between the BHom and $\mathrm{SN}_{1}$, as shown in Fig. 4(b2-b3), which is consistent with the coexistence region (blank) lower to the $\mathrm{SNHO}_{1}$ in Fig. 3(a). The BHom bifurcation is right to $\mathrm{FN}$ point, i.e., the transition point between the stable focus (bold blue curve) and the stable node (thin blue curve). That is to say, the steady state left to and close to the BHom bifurcation corresponds to the stable node. Other symbols and plots are the same as those shown in Fig. 4(a1-a3) such as $\mathrm{SupH}_{2}$, which is not provided in the following figures due to such a bifurcation is not the focus of the present paper.
The results of Fig. 4(a3) and Fig. 4(b3) show that the $\mathrm{SNHO}_{1}$ is responsible for the bifurcation transition from SNIC to BHom and the emergence of SN, which are consistent with the result shown in Fig. 2 or $3(\mathrm{a})$.

Fig. 5 describes the dynamical behavior of the big homoclinic orbit (black) in the phase plane, which corresponds to the BHom bifurcation (at black arrow, $I_{\mathrm{BHom}} \approx$ $3.5204736 \mu \mathrm{A} / \mathrm{cm}^{2}$ ) depicted in Fig. 4(b1-b3). Here the red line and blue line are for the nullclines $d V / d t=0$ and $d n / d t=0$, respectively, as depicted in Fig. 5(a). The intersection between both nullclines form three equilibrium points, a stable node (orange point), a saddle (half-filled point), and an unstable focus (hollow point), which correspond to the LB, MB, and UB in Fig. 4(b1b3), respectively. The position relationship between the stable node and saddle is clearly shown in the insert figure of Fig. 5(a) and Fig. 5(c). Superposing the phase 

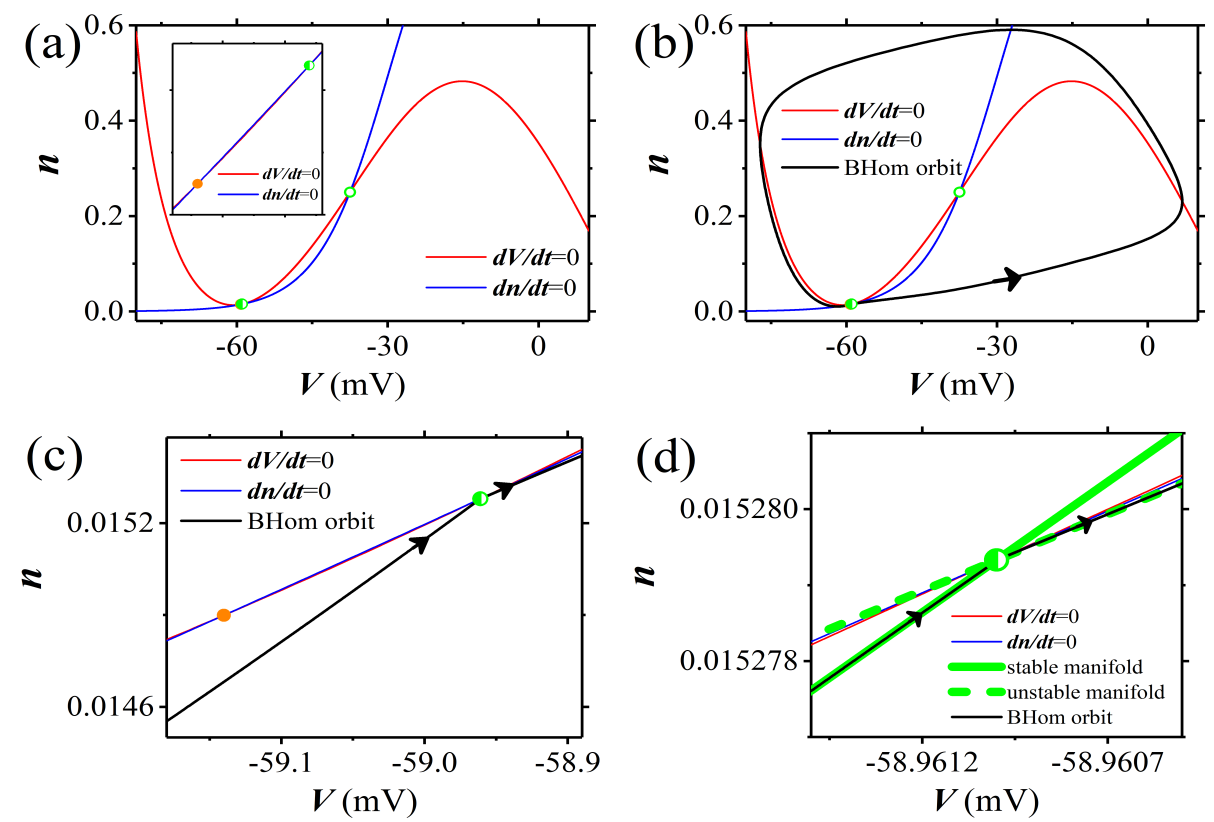

Fig. 5: The dynamics related to the big homoclinic (BHom) orbit (black) at $I_{\mathrm{BHom}} \approx 3.5204736 \mu \mathrm{A} / \mathrm{cm}^{2}$ and $V_{1 / 2 \_n}=-29.8 \mathrm{mV}$ (corresponding to Fig. 4(b1-b3)). (a)The $V$-nullcline $d V / d t=0$ (red) and $n$-nullcline $d n / d t=0$ (blue). Their intersection points are stable node (solid circle), saddle (half-filled circle), and unstable focus (hollow circle); (b) The big homoclinic orbit (BHom, black) with the nullclines; (c) The enlargement of (b) around the stable node and saddle point; (d) The further enlargement of (b) around saddle. The solid and dashed green line represent the stable and unstable manifold of the saddle, respectively. The black arrow indicates the direction for both the trajectory and manifolds of saddle.

trajectory of the BHom orbit onto Fig. 5(a) forms Fig. $5(\mathrm{~b})$ and the details around the saddle are enlarged in Fig. 5(c). The black circle, i.e., the BHom orbit, goes through the saddle (half-filled point) to form a big loop surrounding the stable node (orange point) with anticlockwise (black arrow). The minimal membrane potential of the BHom orbit is lower than that of the stable node. Furthermore, the details of the homoclinic orbit near the saddle are enlarged again in Fig. 5(d), where the solid (dashed) green line represents the stable (unstable) manifold of the saddle. After starting from the saddle, the trajectory of the orbit runs along the unstable manifold (dashed green) of the saddle to form the action potential. Before returning to the saddle, the trajectory of the BHom orbit runs along the stable manifold (solid green line) of the saddle. However, for a common homoclinic orbit, or called small homoclinic (SHom) orbit, the node is outside of the SHom orbit, i.e. the minimal membrane potential of the SHom orbit is higher than that of the node.

Shown in Fig. 6(a) is the change of firing frequency near the SNIC $_{1}$ bifurcation in Fig. 4(a2-a3), where the transition between the stable equilibrium (steady state) and stable limit cycle (spiking) occurs at the $\mathrm{SNIC}_{1}$.
With increasing $I$, the stable steady state changes to spiking via $\mathrm{SNIC}_{1}$ bifurcation and with nearly zero frequency, as shown by the red right arrow in Fig. 6(a). With decreasing $I$, the spiking changes to stable steady state also via $\mathrm{SNIC}_{1}$ bifurcation and with nearly zero frequency, as shown by the down arrow in Fig. 6(a). Therefore, both the spiking and excitability belong to type I at $V_{1 / 2 \_n}=-29$. The types of (spiking, excitability) are labeled as (I, I). Shown in Fig. 6(b) is the change of the firing frequency near the BHom bifurcation depicted in Fig. 4(b2-b3). It can be found that the transition from spiking to steady state is via the BHom bifurcation and exhibits nearly zero frequency, as shown by the red arrow in Fig. 6(b). However, the transition from resting state to spiking occurs at the $\mathrm{SN}_{1}$ bifurcation and manifests nonzero frequency, as shown by the green arrow in Fig. 6(b). Then it follows that the types of (spiking, excitability) are (II, I) at $V_{1 / 2 \_n}=-29.8$. Therefore, with decreasing $V_{1 / 2 \_n}$ from upper side to lower side of $\mathrm{SNHO}_{1}$, the spiking remains unchanged and belongs to type I, whereas the excitability changes from type I to II. 

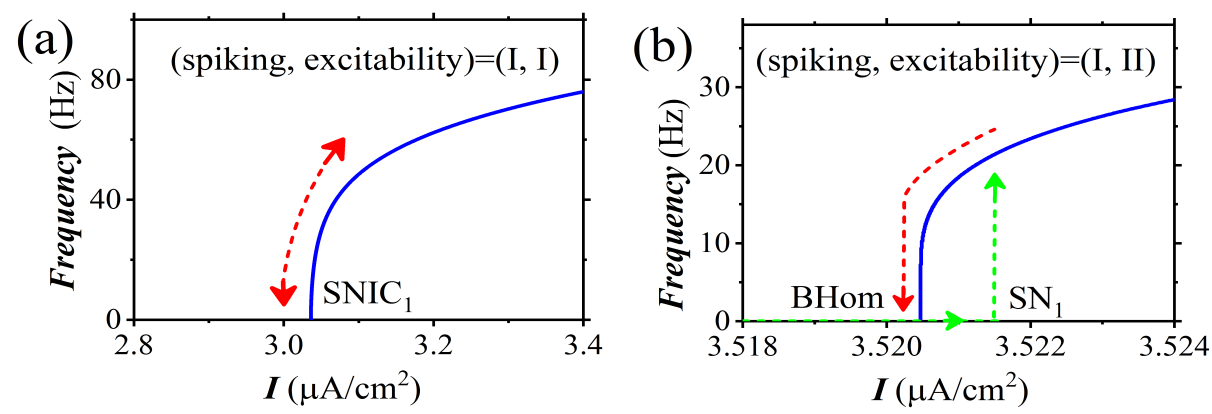

Fig. 6: The change of firing frequency with respect to $I$ at different $V_{1 / 2 \_n}$ values. (a) $V_{1 / 2 \_n}=-29 \mathrm{mV}$ (corresponding to Fig. 4(a2-a3)); (b) $V_{1 / 2 \_n}=-29.8 \mathrm{mV}$ (corresponding to Fig. 4(b2-b3)).
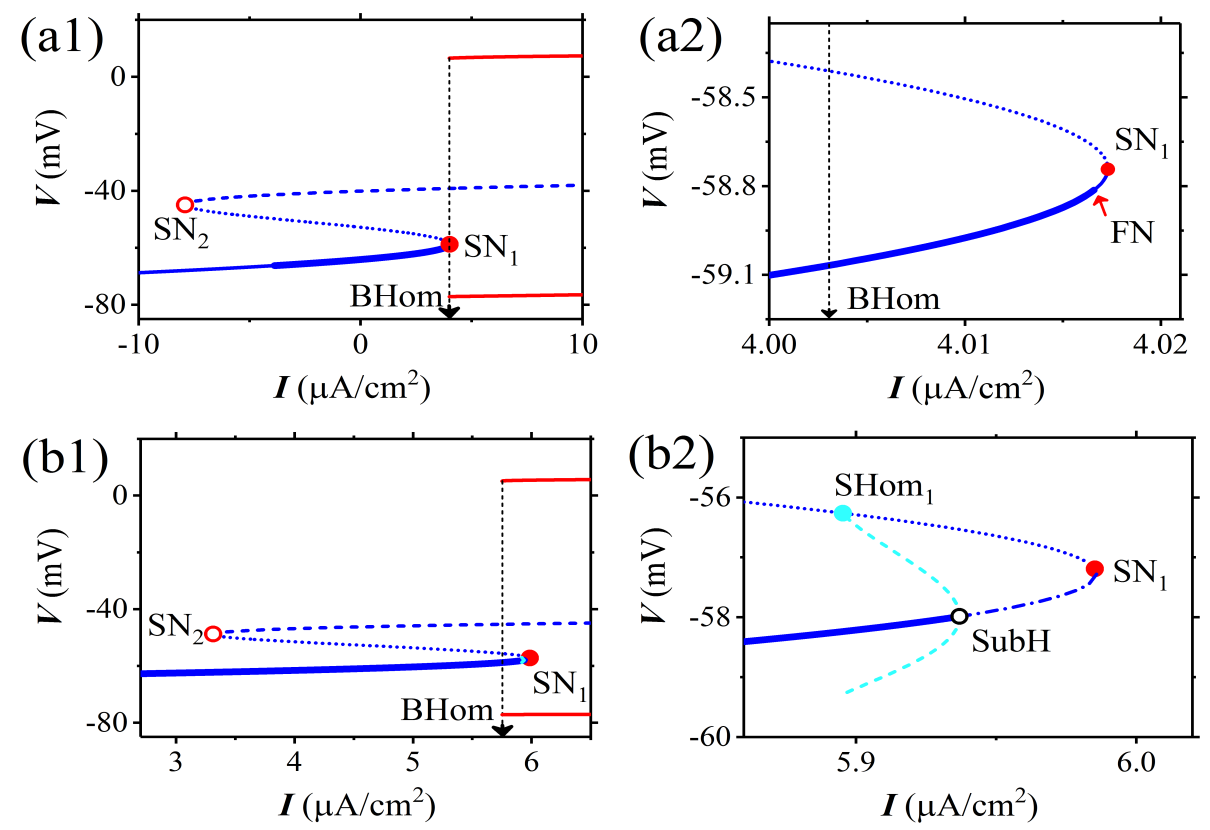

Fig. 7: The one-parameter bifurcation diagram to explain the dynamics around the BT point in Fig. 2(b) or Fig. 3(b). The BHom bifurcation occurs at the black arrow (dashed vertical line). The LB exhibits stable focus (bold) around the BHom bifurcation point. (a1) and its enlargement (a2) correspond to $V_{1 / 2 \_n}=-30.5 \mathrm{mV}$. (b1) and its enlargement (b2) correspond to $V_{1 / 2 \_n}=-32.5 \mathrm{mV}$. A sub-critical Hopf (SubH, black hollow point) bifurcation appears on the LB. The unstable limit cycle (cyan dashed line) contacts with the saddle on the MB to form another homoclinic orbit bifurcation ( $\mathrm{SHom}_{1}$, cyan point). Other plots and symbols are same with those in Fig. 4.

\subsubsection{One-parameter bifurcation diagrams around BT}

Fig. 7(a1) corresponds to the one-parameter bifurcation diagrams at $V_{1 / 2 \_n}=-30.5 \mathrm{mV}$ (upper to BT) and it$\mathrm{s}$ enlargement around $\mathrm{SN}_{1}$ is further depicted in Fig. $7(\mathrm{a} 2)$, where the bifurcation structure and symbols are similar to those in Fig. 4(b2-b3). The only difference is that the BHom bifurcation point changes to locate left to the FN point, which means that it is stable focus (bold blue) close to the BHom bifurcation, as shown in Fig. 7(a2). The steady state left to the BHom becomes stable focus instead of stable node in Fig. 4(b3). As
$V_{1 / 2 \_n}$ further decreases to $-32.5 \mathrm{mV}$ (lower to BT), the BHom, $\mathrm{SN}_{1}$, and $\mathrm{SN}_{2}$ still exist, as depicted in Fig. 7(b1). The obvious change is that the equilibrium point on LB exhibits a sub-critical Hopf (SubH, black hollow point) bifurcation and the unstable limit cycle (cyan dashed line) contacts with the saddle on the MB to form another homoclinic orbit bifurcation (labeled as $\mathrm{SHom}_{1}$, cyan point), as shown in Fig. 7(b2), which represents the details of Fig. $7(\mathrm{~b} 1)$ around $\mathrm{SN}_{1}$. Here the bifurcation points $\mathrm{SubH}$ and $\mathrm{SHom}_{1}$ correspond to the codimension-1 bifurcation curves SubH (black dashed) and SHom $_{1}$ (cyan dashed) in Fig. 3(b) respectively. 
Moreover, one sees clearly the coexistence for stable limit cycle and stable equilibrium locates between BHom and $\mathrm{SN}_{1}$ in Fig. 7(a1-a2), whereas between BHom and SubH in Fig. 7(b1-b2). These results are consistent with those observed in Fig. 3(b).

Shown in Fig. 8 is the change of firing frequency at $V_{1 / 2 \_n}=-32.5 \mathrm{mV}$ (corresponding to Fig. 7(b1-b2)). One clearly sees that the transition from spiking to resting occurs at BHom with nearly zero firing frequency, as indicated by the red arrow. Therefore the type of spiking still corresponds to BHom and belongs to type I. Moreover, as indicated by the green arrow, it is via SubH bifurcation with a nearly fixed nonzero value that the resting changes to spiking, which thus shows type II excitability. Therefore, at $V_{1 / 2 \_n}=-32.5$ (lower to $\mathrm{BT}$ ), the types of (spiking, excitability) are (I, II). Note that the types of (spiking, excitability) are also (I, II) in Fig. 6(b) $\left(V_{1 / 2 \_n}=-30.5\right.$, upper to BT). Then one has that neither the spiking nor the excitability changes with $V_{1 / 2 \_n}$ decreasing via BT, although the BT bifurcation induces the bifurcation related to equilibrium point changed from the $\mathrm{SN}_{1}$ to the $\mathrm{SubH}$.

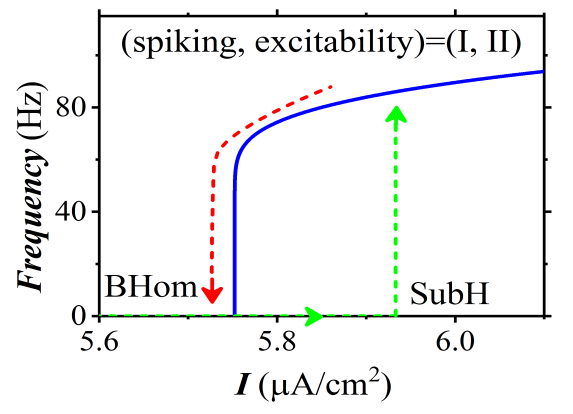

Fig. 8: The change of firing frequency with respect to $I$ at $V_{1 / 2 \_n}=-32.5 \mathrm{mV}$, which corresponds to Fig. 7 (b1-b2).

\subsubsection{One-parameter bifurcation diagrams around $P_{1}$ : the spiking type transition}

The dynamics around degenerate point $\mathrm{P}_{1}$ (Fig. 3(c)) can be explained by the one-parameter bifurcation diagrams shown in Fig. 9, where Fig. 9(a) corresponds to $V_{1 / 2 \_n}=-33.2 \mathrm{mV}$ (upper to point $\mathrm{P}_{1}$ ), Fig. 9(b) to $V_{1 / 2 \_n}=-33.30452 \mathrm{mV}$ (corresponding to point $\mathrm{P}_{1}$ ), and Fig. 9(c) to $V_{1 / 2 \_n}=-33.3 \mathrm{mV}$ (lower to point $\mathrm{P}_{1}$ ). The LPC (magenta solid circle) bifurcation in Fig. 9(c) denotes the fold bifurcation of stable limit cycle, which means that, as $I$ decreases, the stable limit cycle (red curves) and unstable limit cycle (orange dashed curves) coalesce and then disappear via LPC. The insert figure
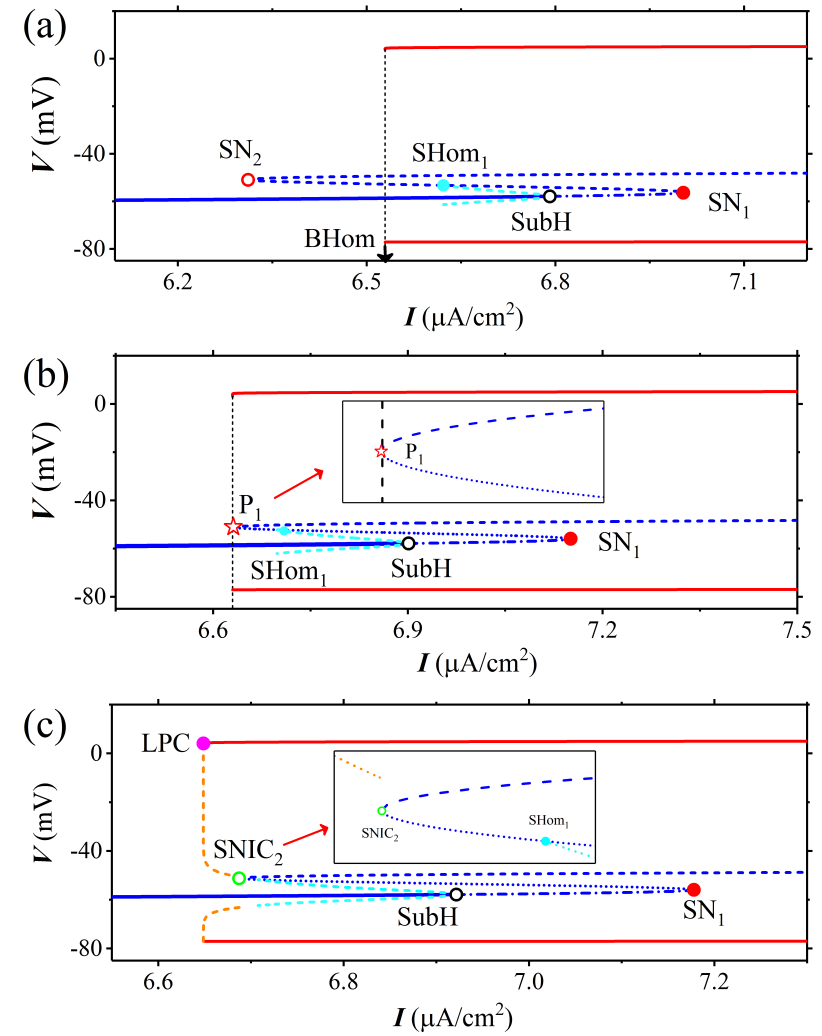

Fig. 9: The one-parameter bifurcation with respect to $I$ at different $V_{1 / 2 \_n}$ values around the degenerate bifurcation point $\mathrm{P}_{1}$ in Fig. 3(c). Shown in dotted blue is the $\mathrm{MB}$ for the saddle and in dashed blue is the UB for the unstable focus. The orange (cyan) dashed line is for the unstable limit cycle. (a) $V_{1 / 2 \_n}=-33.2 \mathrm{mV}$. The points $\mathrm{SHom}_{1}$ (cyan) and $\mathrm{SHom}_{2}$ (orange) are for the saddle homoclinic orbit bifurcation; (b) $V_{1 / 2 \_n}=-33.2845$ $\mathrm{mV}$. The point $\mathrm{P}_{1}$ (red hollow star) corresponds to that in Fig. $3(\mathrm{c})$; (c) $V_{1 / 2 \_n}=-33.3 \mathrm{mV}$. The LPC (magenta solid circle) denotes the fold bifurcation of limit cycle and is formed by the intersection of stable limit cycle (red solid curves) and unstable limit cycle (orange dashed curves). The $\mathrm{SNIC}_{2}$ (green hollow circle) denotes a SNIC bifurcation and is formed by that the disappearance of unstable limit cycle (orange dashed curves) occurs at the intersection of UB and MB. Other plots and symbols are the same as those in Figs. 4 and 7 .

in Fig. 9(c) shows that the unstable limit cycle (orange dashed line) disappears at the intersection point of UB and MB, which thus denotes a SNIC bifurcation (labeled as $\mathrm{SNIC}_{2}$ ). Other plots in Fig. 9 are the same as those in Fig. 7(b1-b2). Here the bifurcation points LPC and $\mathrm{SNIC}_{2}$ correspond to the codimension-1 bifurcation curves LPC (magenta) and $\mathrm{SNIC}_{2}$ (green dashed) 
in Fig. 3(c). The stable limit cycle (red curves) in Fig. 9(a) terminates via the BHom bifurcation, in Fig. 9(b) via the red star (the intersection of $\mathrm{UB}$ and $\mathrm{MB}$ ), in Fig. 9(c) via the LPC bifurcation (magenta solid circle). Therefore, the red star in Fig. 9(b) corresponds to the point $\mathrm{P}_{1}$ in Fig. 3(c) and represents the transition from BHom (Fig. 9(a)) to LPC (Fig. 9(c)) with decreasing $V_{1 / 2 \_n}$. Meanwhile, via such point $\mathrm{P}_{1}, \mathrm{SN}_{2}$ in Fig. 9(a) changes to $\mathrm{SNIC}_{2}$ in Fig. 9(c). Besides, the coexistence behavior locates between BHom and $\mathrm{SubH}$ in Fig. 9(a) and between LPC and SubH in Fig. 9(c). These results are consistent with those observed in Fig. $3(\mathrm{c})$.

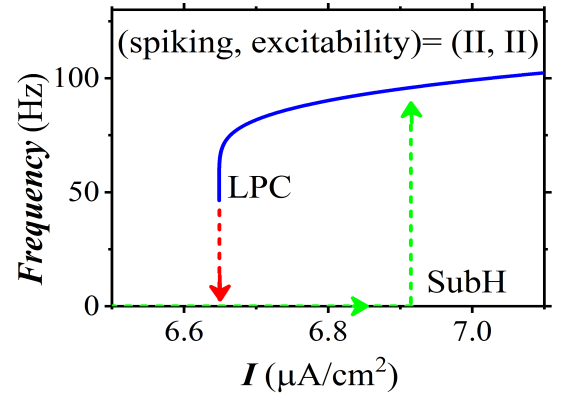

Fig. 10: The change of firing frequency with respect to $I$ at $V_{1 / 2 \_n}=-33.3 \mathrm{mV}$, which corresponds to Fig. $9(\mathrm{c})$.

Fig. 10 represents the change of firing frequency at $V_{1 / 2 \_n}=-33.3 \mathrm{mV}$ (lower to point $\mathrm{P}_{1}$ ). The type of spiking now is determined by the firing frequency near the LPC bifurcation, which exhibits a nearly fixed, nonzero value. That is to say, the spiking belongs to type II. Besides, the excitability is still type II corresponding to the SubH bifurcation. Therefore, for $V_{1 / 2 \_n}=-33.3$ $\mathrm{mV}$ (lower to point $\mathrm{P}_{1}$ ), types of (spiking, excitability) are (II,II). Note that the types of (spiking, excitability) at $V_{1 / 2 \_n}=-32.5$ (upper to point $\mathrm{P}_{1}$ ) are (I,II), as depicted in Fig. 8. That is to say, as $V_{1 / 2 \_n}$ decreases via the point $\mathrm{P}_{1}$, the spiking changes from type I to type II, meanwhile the excitability type keeps unchanged and still belongs to II. The results show that the degenerate bifurcation point $\mathrm{P}_{1}$ plays an important role in modulating spiking between type I and type II.

\subsubsection{One-parameter bifurcation diagrams around points $\mathrm{N}$ and $\mathrm{SNHO}_{2}$}

To further show the dynamics near the degenerate bifurcation point $\mathrm{N}$ in Fig. 3(d), one-parameter bifurcation diagrams with respect to $I$ at different $V_{1 / 2 \_n}$ values around the point $\mathrm{N}$ are shown in Fig. 11, which
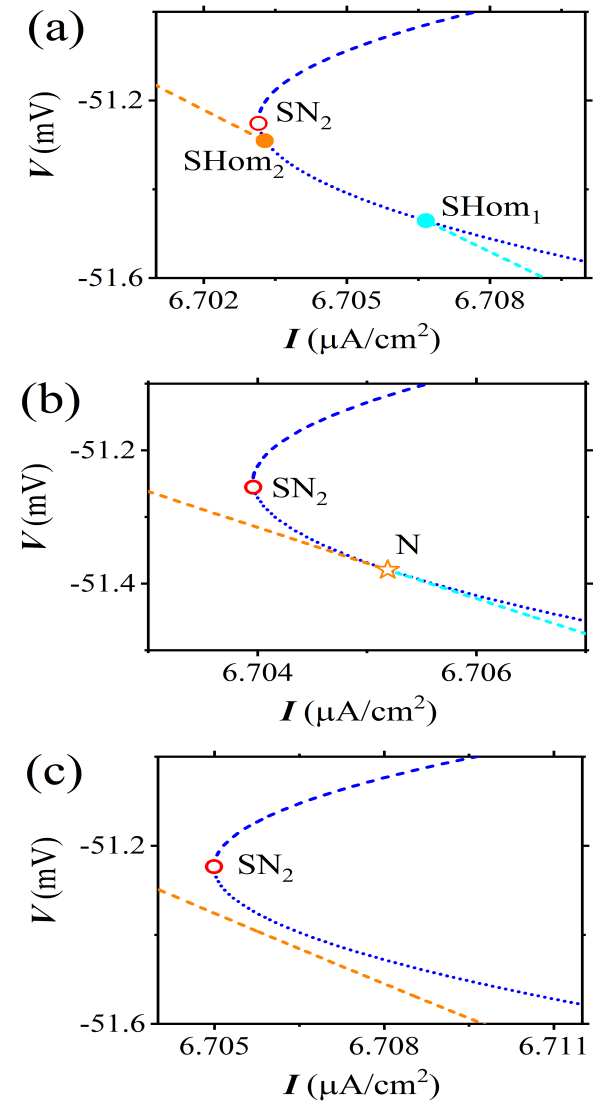

Fig. 11: The one-parameter bifurcation with respect to $I$ at different $V_{1 / 2 \_n}$ values around the degenerate bifurcation point $\mathrm{N}$ in Fig. 3(d). Shown in dotted blue is the MB for the saddle and in dashed blue is the UB for the unstable focus. The orange (cyan) dashed line is for the unstable limit cycle. (a) $V_{1 / 2 \_n}=-33.3043$ $\mathrm{mV}$. The points $\mathrm{SHom}_{1}$ (cyan) and $\mathrm{SHom}_{2}$ (orange) are for the saddle homoclinic orbit bifurcation; (b) $V_{1 / 2 \_n}=-33.30452 \mathrm{mV}$. The degenerate bifurcation point $\mathrm{N}$ (orange hollow star) corresponding to that in Fig. 3(d); (c) $V_{1 / 2 \_n}=-33.3048 \mathrm{mV}$. Both the $\mathrm{SHom}_{2}$ and the $\mathrm{SHom}_{1}$ vanish.

represents the local view of the bifurcation structure around the intersection of the MB and UB of equilibrium (blue) curve. When $V_{1 / 2 \_n}=-33.3043 \mathrm{mV}$ (upper to $\mathrm{N}$ ), the $\mathrm{SHom}_{2}$ (orange point) locates left to the $\mathrm{SHom}_{1}$ (cyan point), as shown in Fig. 11(a). Here $\mathrm{SHom}_{2}$ denotes a homoclinic orbit bifurcation, which is formed by the unstable limit cycle (orange dashed line) contacting with the saddle on the $\mathrm{MB}$, and corresponds to the codimension-1 bifurcation curve $\mathrm{SHom}_{2}$ (purple dashed) in Fig. 3(d). For $V_{1 / 2 \_n}=-33.30452 \mathrm{mV}$ (at point N), the $\mathrm{SHom}_{2}$ and the SHom 1 contact with each other to form degenerate bifurcation $\mathrm{N}$ (orange hollow star), as shown in Fig. 11(b). When $V_{1 / 2_{-} n}=-33.3048$ 
$\mathrm{mV}$ (lower to point N), both $\mathrm{SHom}_{2}$ and $\mathrm{SHom}_{1}$ disappear, as shown in Fig. 11(c). Therefore, the degenerate point $\mathrm{N}$ describes how the $\mathrm{SHom}_{2}$ and $\mathrm{SHom}_{1}$ coalesce and disappear, as depicted in Fig. 3(d). Since $\mathrm{SHom}_{2}$ and $\mathrm{SHom}_{1}$ are related with unstable limit cycle and unstable equilibrium, point $\mathrm{N}$ has no direct influences on the spiking or excitability transition.

Moreover, one can also explains the dynamics of $\mathrm{SNHO}_{2}$ in Fig. 3(d) from the one-parameter bifurcation diagrams shown in Fig. 9(c) $\left(V_{1 / 2 \_n}=-33.3\right.$, upper to $\left.\mathrm{SNHO}_{2}\right)$ and Fig. 11(a) $\left(V_{1 / 2 \_n}=-33.3043\right.$, lower to $\mathrm{SNHO}_{2}$ ). The bifurcation structures in Fig. 9(c) and Fig. 11(a) are almost identical except for two pieces of difference. One striking difference is that the unstable limit cycle (orange dashed) terminates via the $\mathrm{SNIC}_{2}$ bifurcation in former whereas via $\mathrm{SHom}_{2}$ bifurcation in latter. The other one is that, the intersection of UB and MB denotes SNIC bifurcation (labeled as $\mathrm{SNIC}_{2}$ ) in Fig. 9(c), whereas $\mathrm{SN}$ bifurcation (labeled as $\mathrm{SN}_{2}$ ) in Fig. 11(a). Different from the $\mathrm{SNHO}_{1}$ depicted in Fig. 3(a), the dynamical behaviors shown in Fig. 3(d) related to the 3 bifurcation curves through the $\mathrm{SNHO}_{2}$ are unstable. More precisely to say, the curve $\mathrm{SN}_{2}$ (red dashed) is related to the unstable node, the curve $\mathrm{SNIC}_{2}$ (green dashed) related to the unstable node and unstable limit cycle, as depicted in Fig. 9(c) and Fig. 11(a), respectively, and the curve $\mathrm{SHom}_{2}$ (purple dashed) related to the unstable limit cycle. Therefore, the $\mathrm{SNHO}_{2}$ has no direct influence on the spiking or excitability transitions.

\subsubsection{One-parameter bifurcation diagrams around $G H$}

The one-parameter bifurcation diagrams shown in Fig. 12 can detail the dynamics of Bautin bifurcation (GH, cyan solid circle) in Fig. 2(b). When $V_{1 / 2 \_n}=-34$ $\mathrm{mV}$ (upper to $\mathrm{GH}$ ), it exhibits LPC (magenta solid circle) and SubH (black hollow circle) bifurcation, and the coexistence behavior locates between LPC and Sub$\mathrm{H}$, as depicted in Fig. 12(a). As $V_{1 / 2 \_n}$ decreases to be lower than $\mathrm{GH}$, for example, at $-40 \mathrm{mV}$, it exhibits sup-critical Hopf ( $\mathrm{SupH}_{1}$, black solid circle) bifurcation, and no coexisting behavior appears, as depicted in Fig. 12(b). Here the bifurcation point $\mathrm{SupH}_{1}$ corresponds to the codimension-1 bifurcation curve $\mathrm{SupH}_{1}$ (black solid) in Fig. 2(b). Shown in Fig. 13 is the change of firing frequency at $V_{1 / 2 \_n}=-40$ (corresponding to Fig. $12(\mathrm{~b}))$. The transitions between the steady state and spiking occur both via the $\mathrm{SupH}_{1}$ bifurcation, where the firing frequency is a nearly fixed, non-zero value. Therefore, the types of (spiking, excitability) are still (II, II) at $V_{1 / 2_{n} n}=-40$ (lower to $\mathrm{GH}$ ). The GH point does not induce the transition between excitability type or spiking type when compared with those shown in Fig. $10\left(V_{1 / 2 \_n}=-33.3\right.$, upper to $\left.\mathrm{GH}\right)$.
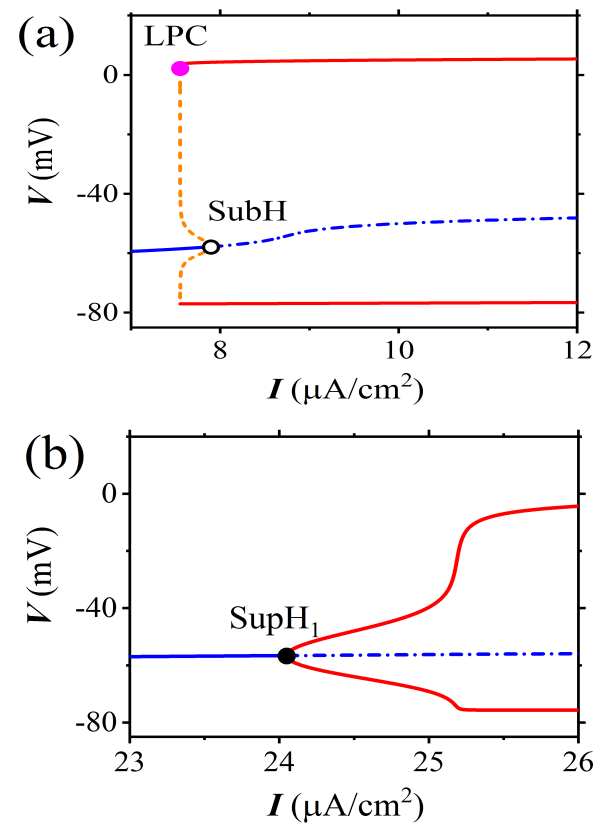

Fig. 12: One-parameter bifurcation diagrams to detail the dynamics of GH in Fig. 2(b). The equilibrium (blue) curve exhibits a monotonic characteristic. (a) $V_{1 / 2 \_n}=-34 \mathrm{mV}$. The stable limit cycle (red solid) and the unstable limit cycle (dashed orange) contacts to form the LPC bifurcation (magenta solid circle). SubH (black hollow circle) denotes the sub-critical Hopf bifurcation; (b) $V_{1 / 2 \_n}=-40 \mathrm{mV}$. SupH $\mathrm{Su}_{1}$ (black solid circle) denotes the sup-critical Hopf bifurcation.

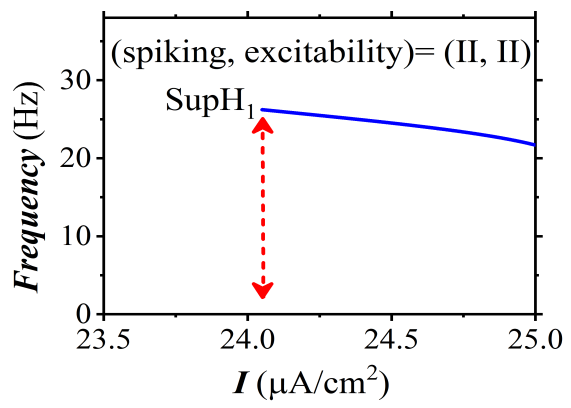

Fig. 13: The change of firing frequency with respect to $I$ at $V_{1 / 2 \_n}=-40 \mathrm{mV}$, which corresponds to Fig. $12(\mathrm{~b})$. 


\subsubsection{One-parameter bifurcation diagrams around CP}

When $V_{1 / 2 \_n}$ is upper to the $\mathrm{CP}$, the equilibrium point curve exhibits a $S$-shaped curve and two SN bifurcation points $\mathrm{SN}_{1}$ and $\mathrm{SN}_{2}$, as depicted in Figs. 4, 7, and 9. When $V_{1 / 2 \_n}$ is lower to the CP, the equilibrium point curve becomes a monotonous characteristic and the SN bifurcation of the equilibrium points disappears, as depicted in Fig. 12. Therefore, the Cusp bifurcation (CP) determines the shape of the equilibrium point curve in $(I, V)$ bifurcation plane, and also corresponds to the disappearance of the $\mathrm{SN}$ bifurcation. The point $\mathrm{CP}$ has nothing to do with the spiking or excitability transitions due to that it is unrelated to the bifurcation of limit cycle.

\subsection{The PIR spike for SNIC, BHom and LPC} bifurcation

\subsubsection{The PIR spike for SNIC}

The negative stimulation can induce spike from the steady state near the SNIC bifurcation depicted in Fig. 4(a1-a3), whereas no spike is evoked near the SNIC for the ML model in Ref. [22]. Therefore, our result presents a novel viewpoint of the post-inhibitory rebound (PIR) spike. Shown in Fig. 14 are the responses (upper panel) of the steady state $\left(I=3.03 \mu \mathrm{A} / \mathrm{cm}^{2}\right)$ to the inhibitory pulse stimulation (lower panel). A negative impulse stimulation with amplitude $A=-115.56$ $\mu \mathrm{A} / \mathrm{cm}^{2}$ (black, lower panel) can induce a PIR spike (black, upper panel) while with amplitude $A=-115.55$ $\mu \mathrm{A} / \mathrm{cm}^{2}$ (red dotted, lower panel) can evoke not a PIR spike but the subthreshold membrane potential (red dotted, upper panel).

The threshold curve for the steady state $n$ ear the SNIC bifurcation The PIR spike or the subthreshold membrane potentials can be explained by the equilibrium points and the threshold dynamics in the phase plane $(V, n)$. Shown in Fig. 15(a) are the nullclines $d V / d t=0$ (red) and $d n / d t=0$ (blue) for $V_{1 / 2 \_n}=-29 \mathrm{mV}$ and $I=3.03 \mu \mathrm{A} / \mathrm{cm}^{2}$ (left and close to $I_{\mathrm{SNIC}} \approx 3.03631 \mu \mathrm{A} / \mathrm{cm}^{2}$ in Fig. $\left.4(\mathrm{a} 2-\mathrm{a} 3)\right)$. Their intersection points denote the stable node (orange solid point), the saddle (half-filled point), and the unstable focus (hollow point). The stable node is a global stable attractor and corresponds to the resting state in Fig. 14. The insert figure shows the details around the stable node and the saddle.

For Eq. (1), the initial value of $(V, n)$ to evoke a spike locates within the blank area, and the initial value to evoke subthreshold membrane potentials within the

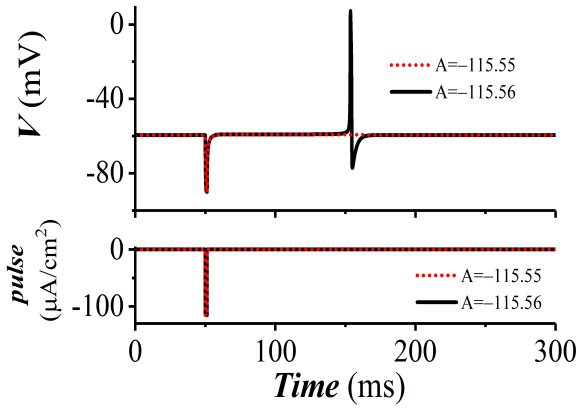

Fig. 14: The response of the steady state to an inhibitory pulse current for $V_{1 / 2 \_n}=-29 \mathrm{mV}$ and $I=3.03$ $\mu \mathrm{A} / \mathrm{cm}^{2}$ (left and close to the SNIC bifurcation in Fig. 4(a2-a3)). The inhibitory pulse current with strength $A=-115.56 \mu \mathrm{A} / \mathrm{cm}^{2}$ (lower panel, black) can evoke a PIR spike (upper panel, black), while $A=-115.55$ $\mu \mathrm{A} / \mathrm{cm}^{2}$ (lower panel, red dotted) can induce not a PIR spike but the subthreshold membrane potential (upper panel, red dotted).

yellow area, as shown in Fig. 15(b1-b3) and Fig. 15(c1c3). The border between the yellow and blank areas is the threshold curve. The square denotes the phase point at the termination time of the inhibitory pulse stimulation.

The trajectory for $A=-115.55 \mu \mathrm{A} / \mathrm{cm}^{2}$, which corresponds to the subthreshold membrane potential (red dotted) in Fig. 14 and is evoked from the stable node (orange solid point), wholly locates within the yellow area, as shown by the red dotted curve in Fig. 15(b1). Its details around the square and saddle are further enlarged in Fig. 15(b2) and Fig. 15(b3) respectively. The trajectory at the termination time (square) of the inhibitory stimulation locates within the yellow area, which shows that the inhibitory stimulation with $A=-115.55 \mu \mathrm{A} / \mathrm{cm}^{2}$ cannot induce the trajectory (red dotted) to run across the threshold curve, as shown in Fig. 15(b2). And the trajectory near the saddle (halffilled point) locates left to the stable manifold (stable green line) of the saddle, i.e. the membrane potential lower than that of the saddle.

However, the trajectory for $A=-115.56 \mu \mathrm{A} / \mathrm{cm}^{2}$, which corresponds to the PIR spike (black) in Fig. 14, exhibits very different dynamics, as shown by the black curve in Fig. 15(c1), (c2), and (c3), where the latter two represent the enlargement of the former one near the square and saddle respectively. The inhibitory stimulation with $A=-115.56 \mu \mathrm{A} / \mathrm{cm}^{2}$ induces the trajectory (black) to run across the down left part of the threshold curve with a negative slope, i.e. go from the yellow area to blank area along the direction from up-right to down-left, as shown in Fig. 15(c2). The trajectory near 

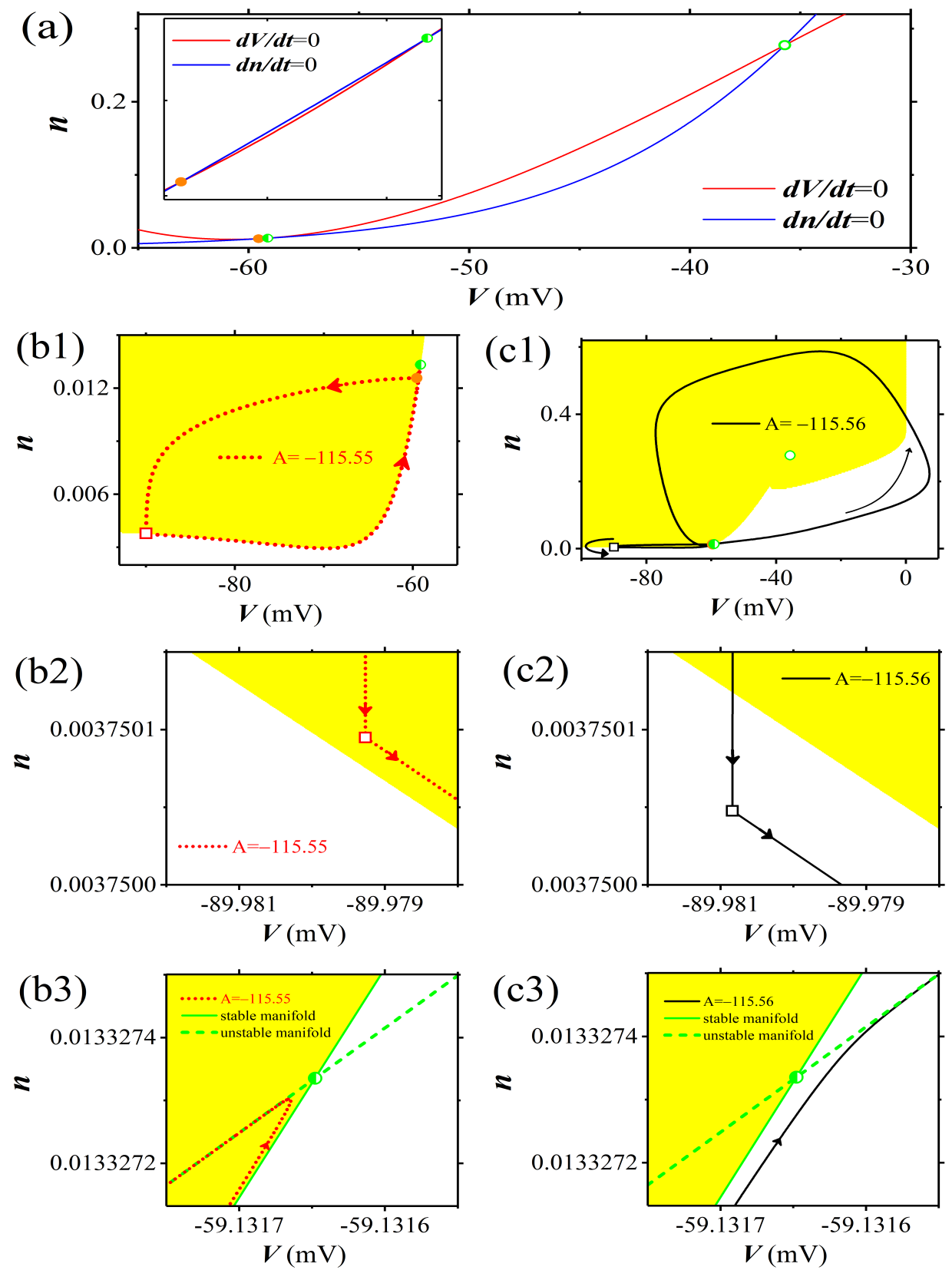

Fig. 15: The dynamics for the PIR spike or the subthreshold membrane potentials shown in Fig. 14. (a) The stable node (orange solid point), saddle (half-filled point), and unstable focus (hollow point) correspond to the intersection points between nullclines $d V / d t=0$ (red) and $d n / d t=0$ (blue). Inset shows the details around the node and saddle; (b1) The phase trajectory (red dotted) for $A=-115.55 \mu \mathrm{A} / \mathrm{cm}^{2}$ corresponding to the subthreshold membrane potential (red dotted) in Fig. 14; (b2) The enlargement of (b1) near the square (i.e., the phase point at the termination of the pulse stimulation); (b3) The enlargement of (b1) near the saddle; (c1) The phase trajectory (black) for $A=-115.56 \mu \mathrm{A} / \mathrm{cm}^{2}$ corresponding to the PIR spike (black) in Fig. 14; (c2) The enlargement of (c1) near the square; (c3) The enlargement of (c1) near saddle. The blank and yellow area represent the collection of the initial values to induce a spike and not a spike but threshold behaviors, respectively. The border between the yellow and blanket areas forms the threshold curve. The arrow indicates the flowing direction of the trajectories. The solid (dashed) green line in (b3) and (c3) denotes the stable (unstable) manifold of saddle. 

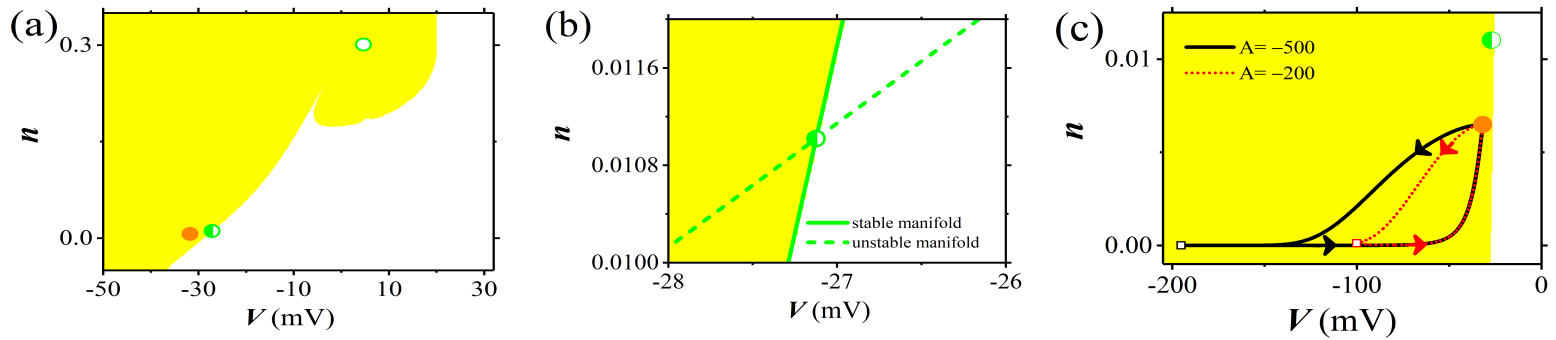

Fig. 16: The threshold curve, phase trajectory evoked by the strong inhibitory pulse stimulation, and equilibrium points near the SNIC in the ML model. (a) The stable node (orange point), saddle (half-filled point), unstable focus (hollow point), and the threshold curve (the boundary between the yellow and blank areas). The blank (yellow) area is the collection of the initial values to induce a spike (not a spike but subthreshold behavior); (b) The enlargement of (a) around the saddle. The solid and dashed curves represent the stable and unstable manifolds of the saddle respectively; (c) The trajectories evoked by stimulations with $A=-200 \mu \mathrm{A} / \mathrm{cm}^{2}$ (red dotted) and $A=-500 \mu \mathrm{A} / \mathrm{cm}^{2}$ (black). Arrow shows the running direction of the trajectory. The square denotes the phase point at the termination time of the stimulation.

the saddle locates right to the stable manifold (green solid line) of the saddle and runs along the unstable manifold (green dashed line) of the saddle, as shown in Fig. 15(c3). Therefore, the right part of unstable manifold takes the role to increase the membrane potential near the saddle. Then the membrane potential further increases to form the PIR spike, as shown in Fig. 15(c1). To sum up, it is the part of the threshold curve downleft to the stable node, which exhibits a negative slope, that is responsible for the PIR spike evoked from the stable node near the SNIC. Once the inhibitory stimulation drives the trajectory to run across such part of the threshold curve at the termination time of the stimulation, the PIR spike is evoked, otherwise not. Besides, the part of the threshold curve near the saddle coincides exactly with the stable manifold (solid green) of the saddle, which shows that the dynamics of saddle plays important roles in the generation of the PIR spike. The shape of the threshold curve for the SNIC in the present paper resembles that of Hopf bifurcation in Ref. [22] to a large extent.

\subsubsection{Different from the traditional viewpoint that no PIR spike for SNIC bifurcation in ML model}

To further show the difference of the threshold curve for the SNIC between the $I_{\mathrm{Na}, \mathrm{p}}+I_{\mathrm{K}}$ model in the present paper and the well-known ML model, some results related to Ref [22] are acquired, as shown in Fig. 16 and Fig. A1 of Appendix. The stable node, the saddle, and the unstable focus are labeled with the orange solid point, half-filled point, and hollow point, respectively, and the threshold curve is the boundary between the yellow and blank areas, as shown in Fig. 16(a). The details near the saddle (solid and dashed curve represen-

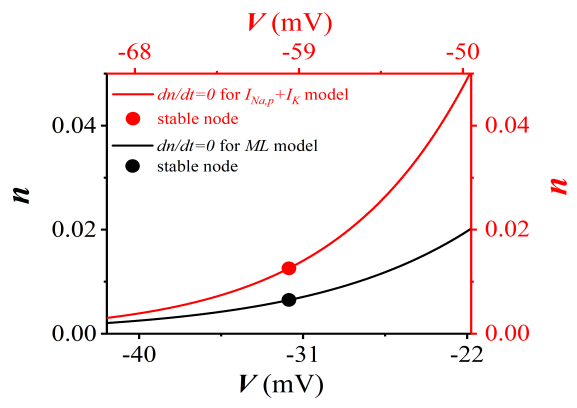

Fig. 17: The $n$-nullclines (solid line) exhibit different slope in phase plane $(V, n)$ for $I_{\mathrm{Na}, \mathrm{p}}+I_{\mathrm{K}}$ model (red)and ML model (black). The solid circle denotes stable node. The parameters are same with those in Fig. 15 and Fig. 16 respectively.

t the stable and unstable manifold of the saddle) are further depicted in Fig. 16(b), which shows that the threshold curve coincides with the stable manifold of the saddle. The whole trajectory evoked by $A=-200$ $\mu \mathrm{A} / \mathrm{cm}^{2}$ (red dotted) and $A=-500 \mu \mathrm{A} / \mathrm{cm}^{2}$ (black) locate in the yellow area, as shown in Fig. 16(c). The square denotes the phase point at the termination time of the stimulation. Even the stimulation is very strong ( $A=-500 \mu \mathrm{A} / \mathrm{cm}^{2}$ ), no PIR spike but subthreshold membrane potential (black) is evoked. Such a result has been widely accepted. The equations, parameter values, bifurcation diagram, and phase portrait of the ML model, please see the Appendix.

Except for the different shapes of threshold curve between $I_{\mathrm{Na}, \mathrm{p}}+I_{\mathrm{K}}$ model and ML model, it can be found that the slope $(d n / d V)$ of $n$-nullcine around the stable node (solid circle) exhibits different slopes, as shown in Fig. 17. The slope of $n$-nullcine for $I_{\mathrm{Na}, \mathrm{p}}+I_{\mathrm{K}}$ 

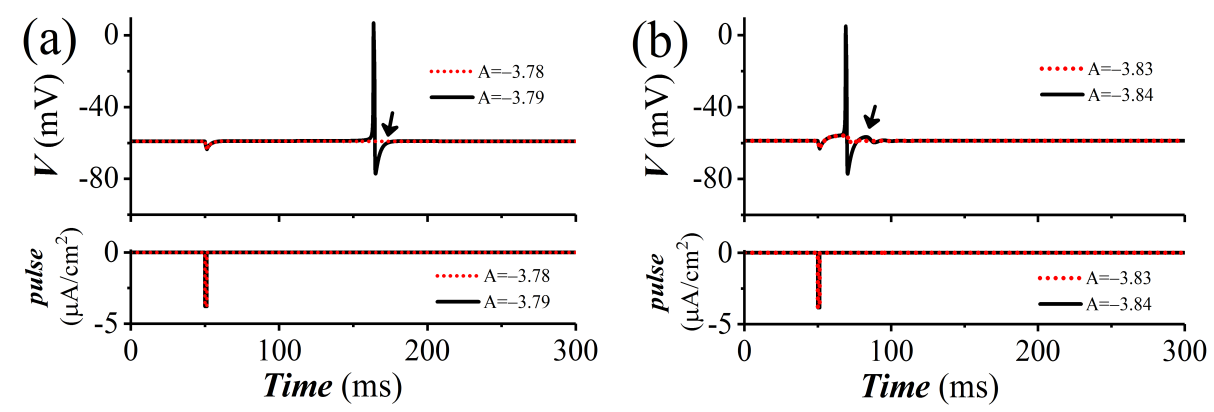

Fig. 18: The PIR spike (black, upper panel) and the subthreshold membrane potentials (red dotted, upper panel) induced by the inhibitory stimulations (lower panel). (a) The resting state (stable node) is near the BHom bifurcation shown in Fig. 4(b1-b3). $A=-3.79 \mu \mathrm{A} / \mathrm{cm}^{2}$ (black) and $A=-3.78 \mu \mathrm{A} / \mathrm{cm}^{2}$ (red dotted) denote the two critical strengths to and not to evoke the PIR spike; (b) The resting state (stable focus) is near BHom bifurcation shown in Fig. 7(b1-b2). The two critical strengths are $A=-3.84 \mu \mathrm{A} / \mathrm{cm}^{2}$ (black) and $A=-3.83$ $\mu \mathrm{A} / \mathrm{cm}^{2}$ (red dotted).
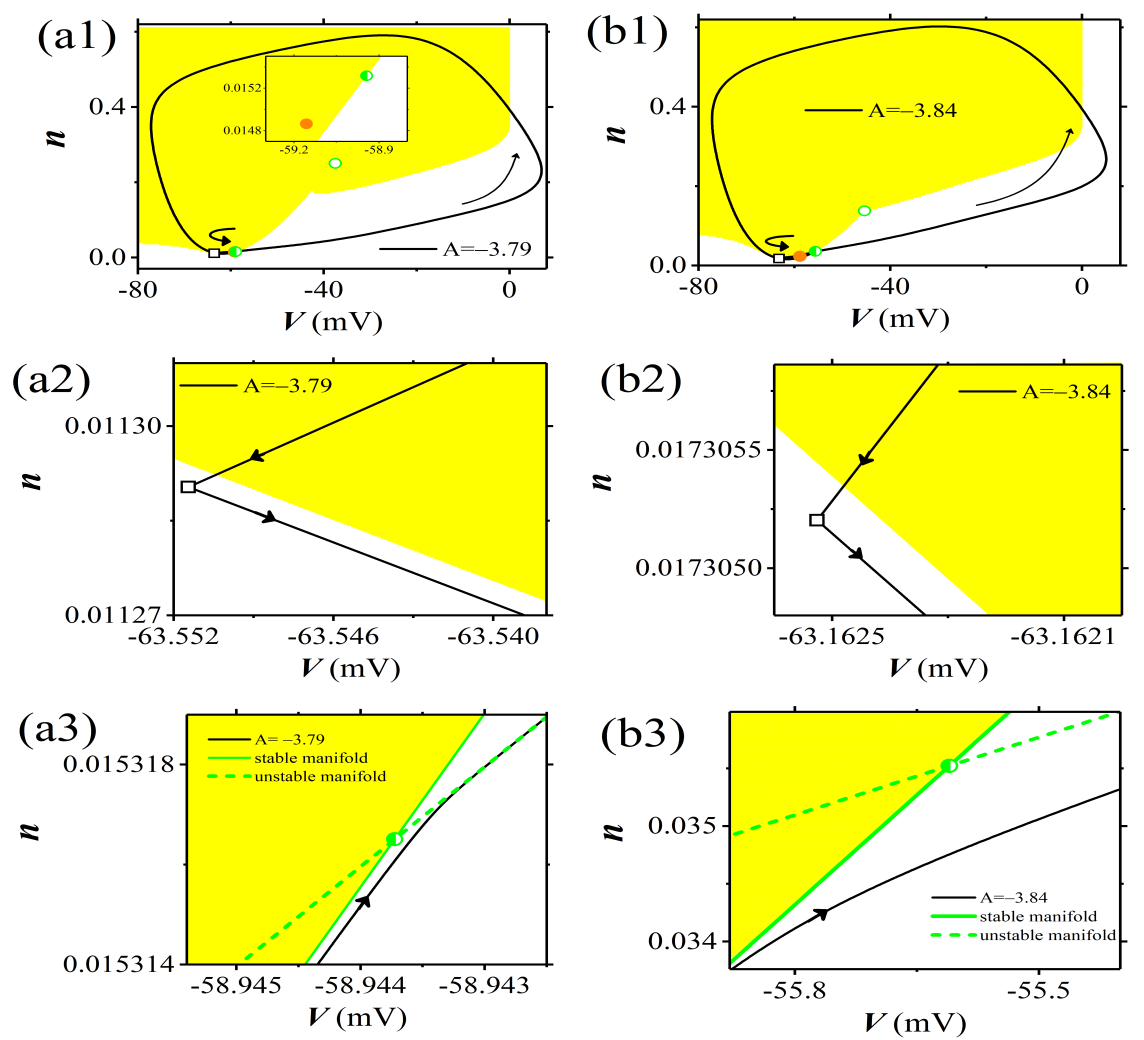

Fig. 19: The dynamics of the PIR spike for the BHom bifurcation shown in Fig. 18. The left panel describes the phase trajectory of the PIR spike in Fig. 18(a) and the right panel corresponds to that in Fig. 18(b). The solid orange point denotes the stable equilibrium, half-filled point for the saddle, and hollow point for the unstable focus. The hollow square represents the phase point at the termination time of the stimulation. The yellow area denotes for the initial values to induce subthreshold behaviors, whereas the blank area for the initial values to induce spike behaviors. Their boundary forms the threshold curve for the spike. (a1)-(b1): The global view of the phase trajectory. Inset in (a1) shows the position relationship of node and saddle; (a2)-(b2): The enlargement of (a1)-(b1) near the squares, respectively; (a3)-(b3): The enlargement of (a1)-(b1) near the saddle, respectively, where the solid (dashed) green line represents the stable (unstable) manifold of the saddle. 
model (red) is larger than that of ML model (black), which influences the vector fields around the $n$-nullcine. The value of slope of $n$-nullcine is a possible important factor to determine the PIR spike or not for a same type of bifurcation, which should be further studied in future.

\subsubsection{The PIR spike near BHom}

Fig. 18 shows the PIR spike evoked from the steady state near the BHom bifurcation. For the stable node at $I=3.52 \mu \mathrm{A} / \mathrm{cm}^{2}$ for $V_{1 / 2 \_n}=-29.8 \mathrm{mV}$, which is left and close to the BHom $\left(I_{\mathrm{BHom}} \approx 3.5204736 \mu \mathrm{A} / \mathrm{cm}^{2}\right)$ shown in Fig. 4(b1-b3), the inhibitory stimulation with strength $A=-3.79 \mu \mathrm{A} / \mathrm{cm}^{2}$ (lower black) can induce PIR spike (upper black), as shown in Fig. 18(a), while the inhibitory stimulation with $A=-3.78 \mu \mathrm{A} / \mathrm{cm}^{2}$ (lower red dotted) induces not PIR spike but subthreshold membrane potentials (upper red dotted).

For the stable focus at $I=5.75 \mu \mathrm{A} / \mathrm{cm}^{2}\left(V_{1 / 2 \_n}=\right.$ $-32.5 \mathrm{mV})$, which is left and close to the BHom $\left(I_{\mathrm{BHom}} \approx\right.$ $5.75239 \mu \mathrm{A} / \mathrm{cm}^{2}$ ) shown in Fig. 7(b1-b2), the inhibitory stimulation with strength $A=-3.84 \mu \mathrm{A} / \mathrm{cm}^{2}$ (lower black) can induce PIR spike (upper black), as shown in Fig. 18(b), while the inhibitory stimulation with $A=$ $-3.83 \mu \mathrm{A} / \mathrm{cm}^{2}$ (lower red dotted) induces the subthreshold membrane potentials (upper red dotted). The membrane potential finally recovers to the steady state. The main difference between Fig. 18(a) and Fig. 18(b) is the absence of damping oscillations for the former and the presence for the latter, as depicted by the black arrows, which is due to that the steady state is stable node for the former and stable focus for the latter.

The phase trajectory for $A=-3.79 \mu \mathrm{A} / \mathrm{cm}^{2}$ is illustrated in left panel of Fig. 19, which correspond to the PIR spike in Fig. 18(a) and is evoked from the stable node. The inset in Fig. 19(a1) shows clearly the position relationship between node (orange point) and saddle (half filled point). Shown in right panel of Fig. 19 is the phase trajectory for $A=-3.84 \mu \mathrm{A} / \mathrm{cm}^{2}$, which correspond to the PIR spike in Fig. 18(b) and is evoked from stable focus (orange point). As depicted in Fig. 19, these two trajectories for PIR spikes related to BHom exhibit dynamics similar to that of the SNIC (Fig. 15). The down-left part of both threshold curves exhibit a negative slope, as shown in Fig. 19(a1) and (b1). The enlargement of the threshold curve with the negative slope near the square (the termination time of the inhibitory stimulation) is shown in Fig. 19(a2) and (b2). The inhibitory stimulation can induce the trajectory run across the threshold curve from up-right to down-left. The enlargement of the trajectory of the PIR spike (black) near the saddle (half-filled point) is shown in Fig. 19(a3) and (b3). The trajectory locates right to the stable manifold (solid green curve) and unstable manifold (dashed green curve), resembling that of the SNIC. The hollow circle in Fig. 19(a1) and Fig. 19(b1) represents the unstable focus.

\subsubsection{The PIR spike near LPC}

Shown in Fig. 20 is the PIR spike near the LPC bifurcation $\left(I_{\mathrm{LPC}} \approx 6.64876 \mu \mathrm{A} / \mathrm{cm}^{2}\right)$ in Fig. 9(c). Fixed $I=$ $6.64 \mu \mathrm{A} / \mathrm{cm}^{2}$ (left and close to LPC bifurcation), the system has a unique equilibrium, i.e., a stable focus (orange point). The inhibitory stimulation with strength $A=-4.1 \mu \mathrm{A} / \mathrm{cm}^{2}$ (black, lower panel) can evoke a PIR spike (black, upper panel) and $A=-4.09 \mu \mathrm{A} / \mathrm{cm}^{2}$ (red dotted, lower panel) induce not PIR spike but subthreshold membrane potential (red dotted, upper panel), as shown in Fig. 20(a). The phase trajectory (black) of the PIR spike exhibits anti-clockwise (black arrow), as depicted in Fig. 20(b). The details around the focus (orange point) and the threshold curve at the square (the termination of the stimulation) are shown in Fig. 20(c) and (d), respectively. The part of the threshold curve locating down-left to the stable focus (orange point) exhibits a negative slope, and the inhibitory stimulation can induce the trajectory run across the threshold curve from up-right to down-left. The square represents the termination time of the stimulation, and damping oscillations appear before recovering to the steady state.

3.5 Summary of the bifurcations underlying the excitability/spiking transition and the PIR spike

In summary, the type I and type II excitabilities are separated by the horizontal line across the codimension-2 bifurcation $\mathrm{SNHO}_{1}$ (red solid point), and type I and type II spiking are separated by the horizontal line across the degenerate bifurcation point $\mathrm{P}_{1}$ (white hollow star), as shown in Fig. 21. Therefore, the plane $\left(I, V_{1 / 2 \_n}\right)$ is divided into 3 regions. From top to bottom, the types of (spiking, excitability) for the three regions are (I, I), (I, II), and (II, II), which are called case-1, -2, and -3 respectively in the present paper. Furthermore, either of case- 2 and case- 3 contains two subcases. The two subcases for case- 2 are separated by the thin line across the codimension- 2 bifurcation BT (black solid point), and are labeled as case-2_1 and case-2_2. And the two subcases for case- 3 are separated by the thin line across the codimension-2 bifurcation GH (cyan solid point), and are labeled as case-3_1 and case-3_2.

The bifurcations related to types of (spiking, excitability) are (SNIC, SNIC), (BHom, $\mathrm{SN}_{1}$ ), (BHom, 

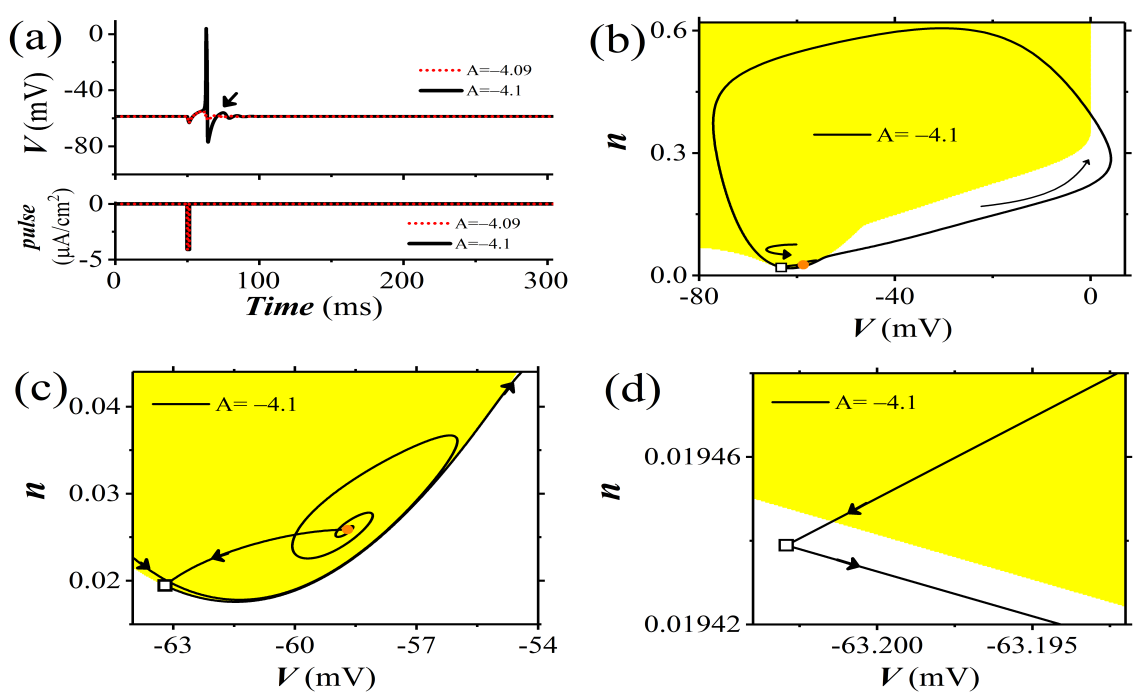

Fig. 20: The PIR spike near the LPC bifurcation in Fig. $9(\mathrm{c})\left(V_{1 / 2 \_n}=-33.3 \mathrm{mV}\right)$. (a) The strength $A=-4.1$ $\mu \mathrm{A} / \mathrm{cm}^{2}$ (black) and $A=-4.09 \mu \mathrm{A} / \mathrm{cm}^{2}$ (red dotted) denote the critical strengths to and not to evoke spike, respectively; (b) The phase trajectory (black) of the PIR spike for $A=-4.1 \mu \mathrm{A} / \mathrm{cm}^{2}$. The solid orange point denotes a stable focus. The square denotes the phase point at the termination time of the stimulation. The yellow (blank) area is for the initial values to induce subthreshold (spike) behaviors. Their boundary forms the threshold curve for the spike; (c) The enlargement of (b) around the focus; (d) The further enlargement of (c) around the square.

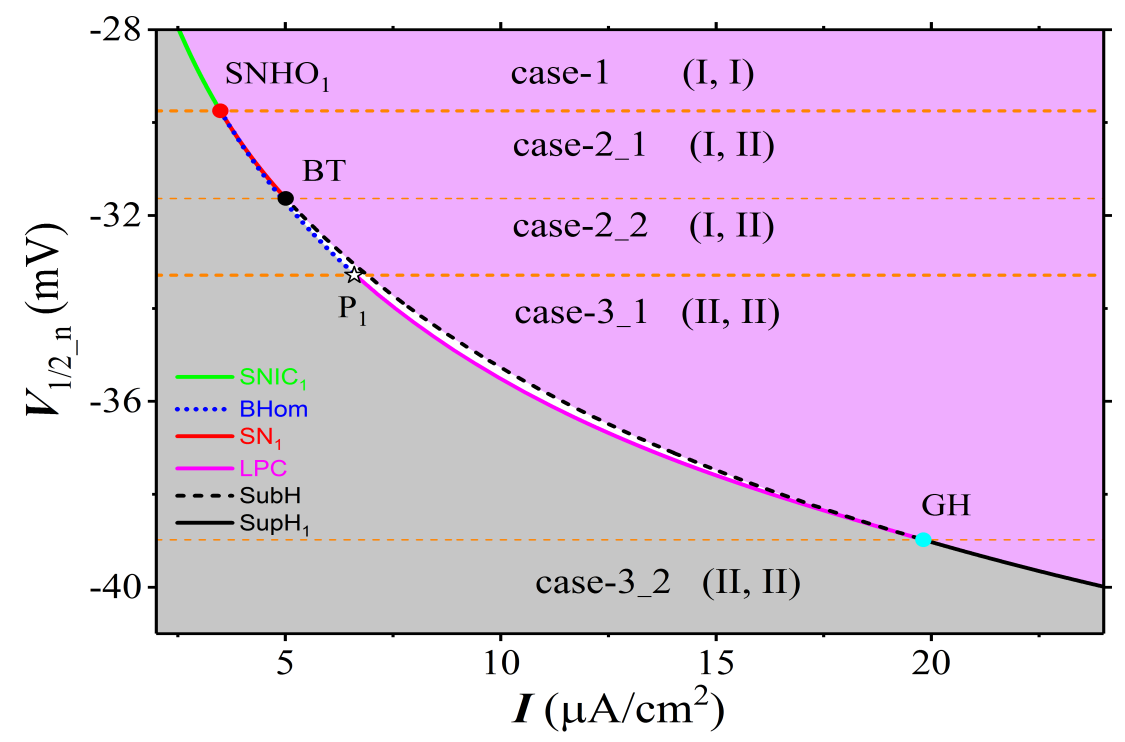

Fig. 21: The relationship between the codimension-2 (or degenerate) bifurcation points and the types of spiking/excitability in two-parameter plane $\left(I, V_{1 / 2} n\right)$. According to the distributions of spiking/excitability type and the related bifurcations, the whole plane is divided into five cases by four dashed horizontal lines, which are labeled with case-1, -2_1, -2_2, -3_1, -3_2, respectively, from top to bottom. According to the neuronal dynamical behaviors, the whole plane is divided into three regions, which are marked with gray (mono-stable equilibrium), pink (stable limit cycle), and blank (coexistence of stable equilibrium and stable limit cycle), respectively. The other plots are same with those shown in Figs. 2-3. 
Table 1: The relationship between the codimension-1 and -2 (or degenerate) bifurcation points and the types of spiking/excitability

\begin{tabular}{|c|c|c|c|c|c|c|}
\hline \multicolumn{2}{|l|}{ Cases } & Behavior & Spiking & Coexistence & Excitability & Behavior \\
\hline \multicolumn{2}{|l|}{ Case-1 } & steady state(PIR) & $\mathrm{SNIC}_{1}(\mathrm{I})$ & No & $\mathrm{SNIC}_{1}(\mathrm{I})$ & Firing \\
\hline \multicolumn{7}{|c|}{ Border: $\mathrm{SHNO}_{1}\left(\mathrm{~V}_{1 / 2 \_n} \approx-29.7491\right)$} \\
\hline \multirow{3}{*}{ Case-2 } & Case-2_1 & steady state (PIR) & $\mathrm{BHom}(\mathrm{I})$ & Yes & $\mathrm{SN}_{1}(\mathrm{II})$ & Firing \\
\hline & Border: $\mathrm{B}$ & $\left(\mathrm{V}_{1 / 2 \_n} \approx-31.6348\right)$ & & & & \\
\hline & Case-2_2 & steady state (PIR) & BHom(I) & Yes & SubH(II) & Firing \\
\hline \multicolumn{7}{|c|}{ Border: $\mathrm{P}_{1}$ point $\left(\mathrm{V}_{1 / 2 \_n} \approx-33.2845\right)$} \\
\hline \multirow{3}{*}{ Case-3 } & Case-3_1 & steady state(PIR) & $\mathrm{LPC}(\mathrm{II})$ & Yes & SubH(II) & Firing \\
\hline & Border:G & $\left(\mathrm{V}_{1 / 2 \_n} \approx-38.9783\right)$ & & & & \\
\hline & Case-3_2 & steady state(not studied) & $\mathrm{SupH}_{1}(\mathrm{II})$ & $\mathrm{No}$ & $\mathrm{SupH}_{1}(\mathrm{II})$ & Firing \\
\hline
\end{tabular}

$\mathrm{SubH}),(\mathrm{LPC}, \mathrm{SubH})$, and $\left(\mathrm{SupH}_{1}, \mathrm{SupH}_{1}\right)$ bifurcations for the case-1, case-2_1, case-2_2, case-3_1, and case-3_2, respectively, as shown in Table 1 and Fig. 21. Near the SNIC for case- 1 and $\mathrm{SupH}_{1}$ for case-3_2, there are no coexistence behaviors, as shown in Table. 1 and Fig. 21. The steady state (gray) and spiking (pink) locate left to and right to the SNIC and $\mathrm{SupH}_{1}$ bifurcation$\mathrm{s}$, respectively. The coexistence of the steady state and spiking (blank area), which lies between the steady state (gray) and spiking (pink), appears between the BHom and $\mathrm{SN}_{1}$ bifurcation for the case-2_1, between the BHom and SubH bifurcations for case-2_2, and between the LPC and SubH bifurcations for case-3_1, as shown in Fig. 21 and Table 1.

For the steady state near SNIC, BHom, and LPC bifurcation points, the PIR spike is identified to be evoked. The PIR spike near the SupH bifurcation is not studied in the present paper due to the small amplitude of the firing, as shown in Fig. 12(b).

\section{Conclusion and Discussion}

Bifurcation has been successfully used to identify dynamics and physiological roles of the neuronal electrical activities. In the present paper, the multiple and complex codimension-1 and codimension- 2 bifurcations underlying the PIR spike induced by the inhibitory stimulation and the transition between type I and type II excitabilities are acquired, which present novel results and comprehensive viewpoints in the following aspects.

Firstly, a novel example for the PIR spike, which appears near the SNIC bifurcation corresponding to type I excitability, is provided, and the speculation that the PIR spike can appear near the BHom bifurcation in Re$\mathrm{f}[22]$ is verified in a two-dimensional neuronal model. The PIR spike appearing near the SNIC bifurcation is a novel result, and the PIR spike near the BHom bifurcation presents a demonstration to the previous spec- ulation. Combined the PIR spike appearing near the LPC bifurcation corresponding to type II excitability and near the Hopf bifurcation in the previous Refs [22, 23,54-56], the comprehensive bifurcations for the PIR spike are acquired. Different from the previous viewpoint that the PIR spike appears near the bifurcation corresponding to type II excitability instead of type I excitability [22], the PIR spike is identified to appear near the SNIC bifurcation related to type I excitability in the present paper, which extends the emerging condition for the PIR spike.

Secondly, a novel threshold curve across which the PIR spike is evoked from the stable node is presented for the SNIC or BHom bifurcations in the $I_{\mathrm{Na}, \mathrm{p}}+I_{\mathrm{K}}$ model, which is different from the threshold curve of the ML model. When the membrane potential is adopted as horizontal ordinate of the phase plane, the striking geometrical feature is that a part of the threshold curve locates down-left to the stable node and exhibits a negative slope, which resembles the threshold curve for the Hopf bifurcation (a part of the threshold curve locates down-left to the stable focus). However, such a part of threshold curve cannot be found for the SNIC bifurcation in the famous ML model.

Last, the comprehensive viewpoint of the codimension2 (or degenerate) bifurcations underlying excitability and spiking transition is presented. Although the $I_{\mathrm{Na}, \mathrm{p}}+$ $I_{\mathrm{K}}$ model is very simple, for example, only two variables and two-dimensional, seven codimension- 2 or degenerate bifurcations and 11 codimension- 1 bifurcation curves are acquired. Type I excitability corresponds to the SNIC bifurcation, while type II excitability corresponds to the SN (right to BHom), SubH (right to BHom or LPC), and SupH bifurcations. Therefore, type I excitability changes to type II excitability via the codimension-2 bifurcation, SNHO, wherein the SNIC bifurcation terminates and meanwhile the SN and BHom bifurcations emerge. Coexistence of the steady s- 
tate and firing appears between the BHom and SN bifurcations, which leads to that the firing frequency at the SN point is non-zero. Therefore, the SN bifurcation corresponds to type II excitability. Type I spiking corresponds to the SNIC and BHom bifurcation points, while type II spiking corresponds to the LPC and SupH bifurcations. Therefore, a degenerate bifurcation point $\mathrm{P}_{1}$, via which the BHom bifurcation terminates and the LPC bifurcation emerges, is responsible for the spiking transition from type I to type II. In addition, the $I_{\mathrm{Na}, \mathrm{p}}+I_{\mathrm{K}}$ model presents bifurcations in two-parameter space more complex than those in the ML model, $\mathrm{H}-$ $\mathrm{H}$ model, and many other neuronal model with highdimension and multiple variables [13,54-58,66-70].

In future, the excitability transition and codimension2 or degenerate bifurcations induced by other parameters or modulations for the $I_{\mathrm{Na}, \mathrm{p}}+I_{\mathrm{K}}$ model, the excitability transition and codimension- 2 or degenerate bifurcations in other neuronal model (such as degenerate points $\mathrm{P}_{2}$ and $\mathrm{c}_{1}$ in Ref. [54]), and the cause for the distinct threshold curves for the PIR spike evoked near the SNIC bifurcation between the ML model and $I_{\mathrm{Na}, \mathrm{p}}+I_{\mathrm{K}}$ model should be further studied, for example, the influences of slope of $n$-nullcline on the different shapes of threshold curve. Through these studies, the deep understanding and comprehensive viewpoints for the excitability transition and PIR spike and the related bifurcations can be acquired, which are helpful for the identification of the nonlinear dynamics and physiological roles of the PIR spike and excitability transition in neuroscience.

\section{Declarrations}

Funding This work was supported by the National Natural Science Foundation of China (Grant Nos. 12072236 , 11872276 and 11762001), the Young Talents of Science and Technology in Universities of Inner Mongolia Autonomous Region (Grant No. NJYT-20-A09), the Postdoctoral Research Project of Henan Province(Grant No. 19030095), and the Key R\&D and Promotion Special Project of Henan Province(Grant No. 212102210543)

Conflicts of interest/Competing interests The authors declare that they have no conflict of interest.

Availability of data and material Data will be made available on reasonable request.

Code availability Not applicable.

Ethics approval Not applicable.

Consent to participate Not applicable.

Consent for publication Not applicable.

\section{References}

1. Braun, H., Wissing, H., Schäfer, K.:Oscillation and noise determine signal transduction in shark multimodal sensory cells, Nature 367,270-273 (1994).

2. Fumiya, T., Genshiro, A., Shoi, S., Etsuo, A., Yukinaga, H., Dimitri, P. et al.: Involvement of $\mathrm{Ca}^{2+}$-dependent hyperpolarization in sleep duration in mammals, Neuron 90, 70-85 (2016).

3. Yang, Y., Cui, Y., Sang, K., Dong, Y., Ni, Z., Ma, S. Hu, $\mathrm{H}$. :Ketamine blocks bursting in the lateral habenula to rapidly relieve depression, Nature 554, 317-322 (2018).

4. Guo, Y., Zhou, P., Yao, Z., et. al.:Biophysical mechanis$\mathrm{m}$ of signal encoding in an auditory neuron, Nonlinear Dyn. 105, 3603-3614 (2021).

5. Glass, L. :Synchronization and rhythmic processes in physiology, Nature 410(6825), 277-284 (2001).

6. Wang, X.J.:Neurophysiological and computational principlesof cortical rhythms in cognition, Physiol. Rev. 90(3), 1195-1268 (2010).

7. Ma, K. H., Gu, H. G., Zhao, Z. G. :Fast-slow variable dissection with two slow variables: A case study on bifurcations underlying bursting for seizure and spreading depression, Int. J. Bifurcation and Chaos (accepted) (2021).

8. Fan, D., Zheng, Y., Yang, Z., Wang, Q. :Improving control effects of absence seizures using single-pulse alternately resetting stimulation (SARS) of corticothalamic circuit, Appl. Math. Mech. -Engl. Ed. 9, 1287-1302 (2020).

9. Xu, Y., Liu, M., Zhu, Z., Ma, J.:Dynamics and coherence resonance in a thermosensitive neuron driven by photocurrent, Chin. Phys. B 29, 098704 (2020).

10. Xie, Y., Xu, J. X., Kang, Y. M., Hu, S. J., Duan, Y. B. : Critical amplitude curves for different periodic stimuli and different dynamical mechanisms of excitability, Commun. Nonlinear Sci. Numer. Simul. 10(7), 823-832 (2005).

11. $\mathrm{Wu}, \mathrm{F} . \mathrm{Q} ., \mathrm{Gu}, \mathrm{H}$. G.:Bifurcations of negative responses to positive feedback current mediated by memristor in neuron model with bursting patterns, Int. J. Bifurcation and Chaos 30(4), 2030009 (2020).

12. Zhang, Y., Xu, Y., Yao, Z., Ma, J.:A feasible neuron for estimating the magnetic field effect, Nonlinear Dyn. 102(3), 1849-1867 (2020).

13. Guan, L. N., Jia, B., Gu, H. G.: A novel threshold across which negative stimulation evokes action potential near a saddle-node bifurcation in a neuronal model with $I_{h}$ current, Int. J. Bifurcation and Chaos 19(14), 1950198 (2019).

14. Lu, L. L., Jia, Y., Ge, M., Xu, Y., Li, A. B.:Inverse stochastic resonance in Hodgkin-Huxley neural system driven by Gaussian and non-Gaussian colored noises, Nonlinear Dyn. 100(1), 877-889 (2020).

15. Zhao, J. Y., Fan, D. G., Wang, Q. S., Wang, Q. Y. : Dynamical transitions of the coupled Class I (II) neurons regulated by an astrocyte, Nonlinear Dyn. 4, 1-12(2021).

16. Zhou, P., Yao, Z., Ma, J., Zhu, Z. G.:A piezoelectric sensing neuron and resonance synchronization between auditory neurons under stimulus, Chaos, Soliton. Fract. 145, 110751 (2021).

17. Song, Z., Zhen, B., Hu, D.: Multiple bifurcations and coexistence in an inertial two-neuron system with multiple delays, Cogn. Neurodyn. 14, 359-374 (2020).

18. Cao, B., Wang, R. X., Gu, H. G., Li, Y. Y.: Coherence resonance for neuronal bursting with spike undershoot, Cogn. Neurodyn. (2020) 15(1), 77-90 (2021). 
19. Yao, Z., Zhou, P., Zhu, Z. G., Ma, J.: Phase synchronization between a light-dependent neuron and a thermosensitive neuron, Neurocomputing 423, 518-534 (2021).

20. Zhang, X. J., Gu, H. G., Guan, L. N.: Stochastic dynamics of conduction failure of action potential along nerve fiber with Hopf bifurcation, Sci. China Technol. Sci. 62(9), 1502-1511 (2019).

21. Hodgkin, A. L.: The local electric changes associated with repetitive action in a non-medullated axon, $J$. Physiol. 107(2), 165-181 (1948).

22. Izhikevich, E. M.: Neural excitability, spiking and bursting, Int. J. Bifurcation and Chaos 10(6), 1171-1266 (2000).

23. Izhikevich, E. M.: Dynamical Systems in Neuroscience: The Geometry of Excitability and Bursting, MIT press, Cambridge(2007).

24. Rinzel, J., Ermentrout, G.: Analysis of neural excitability and oscillations. Chap. 7, 251-291, in: Methods in Neuronal Modeling. MIT Press, Cambridge(1998).

25. Yu, Y. G., Liu, F., Wang, W.: Frequency sensitivity in Hodgkin-Huxley systems, Biol. Cybern. 84, 227-235 (2001).

26. Jia, B., Gu, H. G.: Dynamics and physiological roles of stochastic neural firing patterns near bifurcation points, Int. J. Bifurcation and Chaos 27(7), 17501132017 (2017).

27. Prescott, S. A, De Koninck, Y., Sejnowski, T. J. : Biophysical basis for three distinct dynamical mechanisms of action potential initiation, PLoS Comput. Biol. 4(10), e1000198(2008).

28. Prescott, S. A, Ratté, S., De Koninck, Y., Sejnowski, T. J.: Pyramidal neurons switch from integrators in vitro to resonators under in vivo-like conditions, J. Neurophysiol. 100(6), 3030-3042(2008).

29. Stiefel, K. M., Gutkin, B. S., Sejnowski, T. J.: Cholinergic neuromodulation changes phase response curve shape and type in cortical pyramidal neurons, PLoS One 3, e3947 (2008).

30. Barreiro, A. K., Thilo, E. L., Shea-Brown, E.: A-current and type I/type II transition determine collective spiking from common input, J. Neurophysiol., 108(6), 16311645 (2012).

31. Morozova, E. O, Zakharov, D., Gutkin, B. S., Lapish, C. C., Kuznetsov, A.: Dopamine neurons change the type of excitability in response to stimuli, J. Neurophysiol. 12(12), e1005233(2016).

32. Springer, M. G., Kullmann, P. H., Horn, J. P.: Virtual leak channels modulate firing dynamics and synaptic integration in rat sympathetic neurons: implications for ganglionic transmission in vivo, J. Physiol., 593(4), 803$823(2015)$.

33. Yang, J., Hu, S., Li, F., Xing, J.: Resonance characteristic and its ionic basis of rat mesencephalic trigeminal neurons, Brain Res. 1596, 1-12 (2015).

34. Graves, A. R., Moore, S. J., Spruston, N., Tryba, A. K., Kaczorowski, C. C.: Brain-derived neurotrophic factor differentially modulates excitability of two classes of hippocampal output neurons, J. Neurophysiol. 116(2), 466-471 (2016)

35. Heitmann, S., Rule, M., Truccolo, W., Ermentrout, B.: Optogenetic stimulation shifts the excitability of cerebral cortex from type I to type II: oscillation onset and wave propagation, PLoS Comput. Biol. 13(1), e1005349 (2017).

36. Xie, R. G, Chu, W. G., Hu, S. J., Luo, C.: Characterization of different types of excitability in large somatosensory neurons and its plastic changes in pathological pain states, Int. J. Mol. Sci. 19(1), 161 (2018).
37. Mishra, P., Narayanan. R.: Heterogeneities in intrinsic excitability and frequency-dependent response properties of granule cells across the blades of the rat dentate gyrus, J. Neurophysiol. 123(2), 755-772(2020).

38. Gutkin, B. S., Ermentrout, G. B.: Dynamics of membrane excitability determine interspike interval variability: a link between spike generation mechanisms and cortical spike train statistics, Neural Comput. 10(5), 1047-1065 (1998).

39. Tateno, T., Pakdaman, K.: Random dynamics of the Morris-Lecar neural model, Chaos 14(3), 511$530(2004)$.

40. Ermentout, B.: Type I membranes, phase resetting curves and sychrony, Neural Comput. 8(5), 979-1001 (1996).

41. Hansel, D., Mato, G., Meunier, C.: Synchrony in excitatory neural networks, Neural Comput. $\quad \mathbf{7 ( 2 )}, 307-$ 331(1995).

42. Smeal, R. M., Ermentrout, G. B., White, J. A.: Phaseresponse curves and synchronized neural networks, Philos. Trans. R. Soc. B Biol. Sci. 365(1551), 24072422(2010)

43. Bertrand, S., Cazalets, J.: Postinhibitory rebound during locomotor-like activity in neonatal rat motoneurons in vitro, J. Neurophysiol. 79, 342-351 (1998).

44. Matsushima, T., Tegner, J., Hill, R., Grillner, S.: Gabab receptor activation causes a depression of low- and high-voltage-activated $\mathrm{Ca}^{2+}$ currents, postinhibitory rebound, and postspike afterhyperpolarization in lamprey neurons, J. Neurophysiol. 70, 2606-2619 (1993).

45. Arshavsky, Y., Deliagina, T., Orlovsky, G., Panchin, Y., Popova, L., Sadreyev, R.: Analysis of the central pattern generator for swimming in the mollusk clione, Ann. NY. Acad. Sci. 860, 51-59 (1998).

46. Goaillard, J., Taylor, A., Pulver, S., Marder, E. : Slow and persistent postinhibitory rebound acts as an intrinsic short-term memory mechanism, J. Neurosci. 30(13), 4687-4692 (2010).

47. Felix, R., Fridberger, A., Leijon, S., Berrebi, A., Magnusson, A. : Sound rhythms are encoded by postinhibitory rebound spiking in the superior paraolivary nucleus, J. Neurosci. 31(35), 12566-12578 (2011).

48. Ascoli, G., Gasparini, S., Medinilla, V., Migliore, M.: Local control of postinhibitory rebound spiking in CA1 pyramidal neuron dendrites, J. Neurosci. 30, 64346442(2010).

49. Engbers, J., Anderson, D., Tadayonnejad, R., Mehaffey, W., Molineux, M., Turner, R.: Distinct roles for IT and $\mathrm{IH}$ in controlling the frequency and timing of rebound spike responses, J. Physiol. 589, 5391-5413(2011).

50. Pavlov, I., Scimemi, A., Savtchenko, L., Kullmann, D., Walker, M.: $I_{h}$-mediated depolarization enhances the temporal precision of neuronal integration, Nat. Commun. 2, 199(2011).

51. Gastrein, P., Campanac, E., Gasselin, C., Cudmore, R., Bialowas, A., Carlier, E., Fronzaroli-Molinieres, L., Ankri, N., Debanne, D.: The role of hyperpolarizationactivated cationic current in spike time precision and intrinsic resonance in cortical neuronsin vitro, J. Physiol. 589, 3753-3773 (2011).

52. Ferrante, M., Shay, C., Tsuno, Y. Chapman, W. G., Hasselmo, M. : Post-inhibitory rebound spikes in rat medial entorhinal layer II/III principal cells: In vivo, in vitro, and computational modeling characterization, Cereb. Cortex 27, 2111-2125 (2016).

53. Morris, C., Lecar, H.: Voltage oscillations in the barnacle giant muscle fiber, Biophys. J . 35, 193-213 (1981). 
54. Tsumoto, K., Kitajima, H., Yoshinaga, T., Aihara, K., Kawakami, H.: Bifurcations in Morris- Lecar neuron model, Neurocomputing 69, 293-316 (2006).

55. Liu, C., Liu, X., Liu, S.: Bifurcation analysis of a MorrisLecar neuron model, Biol. Cybern. 108(1), 75-84 (2014).

56. Zhao. Z. G., Li, L., Gu, H. G. : Different dynamical behaviors induced by slow excitatory feedback for type II and III excitabilities, Sci. Rep. 10, 3646 (2020).

57. Zhao, Z. G, Li, L., Gu, H. G.: Dynamical mechanism of hyperpolarization-activated non-specific cation current induced resonance and spike-timing precision in a neuronal model, Front. Cell. Neurosci.12(62)(2018)

58. Guan, L. N., Gu, H. G., Zhao. Z. G. : Dynamics of subthreshold and suprathreshold resonance modulated by hyperpolarization-activated cation current in a bursting neuron, Nonlinear Dyn.104577-601(2021) (Accepted)

59. Govaerts, W., Sautois, B.: The onset and extinction of neural spiking: a numerical bifurcation approach, $J$. Comput. Neurosci. 18(3), 265-274 (2005)

60. Xie, Y., Aihara, K., Kang, Y. M. : Change in types of neuronal excitability via bifurcation control, Phys. Rev. E 77, 021917 (2008)

61. Guo, D. Q., Chen, M. M., Perc, M., Wu, S. D. et al.: Firing regulation of fast-spiking interneurons by autaptic inhibition, Europhys. Lett. 114(3), 30001 (2016)

62. Zhao, Z. G., Gu, H. G.: Transitions between classes of neuronal excitability and bifurcations induced by autapse, Sci. Rep. 7, 6760 (2017).

63. Song, X.L., Wang, H.T., Chen, Y.: Autapse-induced firing patterns transitions in the Morris-Lecar neuron model, Nonlinear Dyn. 96(4), 2341-2350 (2019).

64. Xing, M. M., Song, X. L., Yang, Z. Q., Chen, Y.: Bifurcations and excitability in the temperature-sensitive Morris-Lecar neuron, Nonlinear Dyn. 110, 2687-2698 (2020).

65. Fatoyinbo, H., Brown, R. G., Simpson, D., Brunt, B.: Numerical bifurcation analysis of pacemaker dynamic$\mathrm{s}$ in a model of smooth muscle cells, Bull. Math. Biol. 82(7), 95 (2020)

66. Duan, L., Lu, Q.: Codimension-two bifurcation analysis on firing activities in Chay neuron model, Chaos Soliton Fract. 30(5), 1172-1179 (2006)

67. Duan, L., Lu, Q., Wang, Q. : Codimension-two bifurcation analysis on firing activities in Chay neuron model, Neurocomputing 72(1-3), 341-351 (2008)

68. Shigeki, T., Tetsushi, U., Hiroshi, K., Hiroshi, F., Kazuyuki, A.: Bifurcations in two-dimensional Hindmarsh-Rose type model, Chaos 17(3), 985-998 (2011)

69. Liu, X. L., Liu, S. Q.: Bifurcations in two-dimensional Hindmarsh-Rose type model, Nonlinear Dyn. 67(1), 847-857 (2012)

70. Chen, S. S., Cheng, C. Y., Lin, Y. R.: Application of a two-dimensional Hindmarsh-Rose type model for bifurcation analysis, Int. J. Bifurcation and Chaos 23, 1350055 (2013).

71. Ermentrout, B.: Simulating, Analyzing, and Animating Dynamical Systems. A Guide to XPPAUT for Researchers and Students, SIAM, Philadelphia (2002).

\section{Appendix A.}

The Morris-Lecar (ML) model in Refs. $[3,53]$ reads as follows

$$
\left\{\begin{aligned}
C \frac{d V}{d t} & =I+I_{\mathrm{K}}+I_{\mathrm{Ca}}+I_{\mathrm{L}}, \\
\frac{d n}{d t} & =\frac{n_{\infty}(V)-n}{\gamma(V)}
\end{aligned}\right.
$$

where $V$ denotes the membrane potential with unit $\mathrm{mV}$ and $n$ represents the unitless gating variable of $\mathrm{K}^{+}$ionic channel. $C$ is for the capacitance of a neuron and $I$ for a constant current. $I_{\mathrm{K}}=-g_{\mathrm{K}} n\left(V-E_{\mathrm{K}}\right), I_{\mathrm{Ca}}-g_{\mathrm{Ca}} m_{\infty}(V)\left(V-E_{\mathrm{Ca}}\right)$, and $I_{\mathrm{L}}=-g_{\mathrm{L}}\left(V-E_{\mathrm{L}}\right)$ denote the ionic currents of $\mathrm{K}^{+}, \mathrm{Ca}^{2+}$, leakage ions, respectively. The parameters $E_{\mathrm{K}}, E_{\mathrm{Ca}}$, and $E_{\mathrm{L}}$ are the corresponding reversal potentials, and the parameters $g_{\mathrm{K}}, g_{\mathrm{Ca}}$, and $g_{\mathrm{L}}$ are corresponding the maximal conductances. The functions $m_{\infty}$ and $n_{\infty}$ are the steady state of the gating variables $\mathrm{Ca}^{2+}$ and $\mathrm{K}^{+}$, respectively, $\gamma$ is the time factor, where

$$
\left\{\begin{aligned}
m_{\infty}(V) & =\frac{1}{2}\left(1+\tanh \frac{V-V_{1}}{V_{2}}\right), \\
n_{\infty}(V) & =\frac{1}{2}\left(1+\tanh \frac{V-V_{3}}{V_{4}}\right), \\
\gamma(V) & =\left[0.067 \cosh \frac{V-V_{3}}{2 V_{4}}\right]^{-1} .
\end{aligned}\right.
$$

The parameter values are as follows: $V_{1}=-1.2 \mathrm{mV}, V_{2}$ $=18 \mathrm{mV}, V_{3}=12 \mathrm{mV}, V_{4}=17.4 \mathrm{mV}, E_{\mathrm{L}}=-60 \mathrm{mV}, E_{\mathrm{K}}=$ $-84 \mathrm{mV}, E_{\mathrm{Ca}}=120 \mathrm{mV}, g_{\mathrm{L}}=2 \mu \mathrm{S} / \mathrm{cm}^{2}, g_{\mathrm{K}}=8 \mu \mathrm{S} / \mathrm{cm}^{2}$, $g_{\mathrm{Ca}}=4 \mu \mathrm{S} / \mathrm{cm}^{2}$, and $C=20 \mu \mathrm{F} / \mathrm{cm}^{2}$. The parameter $I$ is considered as bifurcation parameter.

The bifurcation diagram for the SNIC bifurcation $\left(I_{\text {SNIC }} \approx\right.$ $\left.39.96 \mu \mathrm{A} / \mathrm{cm}^{2}\right)$ is shown in Fig. A.1(a), and the equilibrium points and nullclines at $I=39.95 \mu \mathrm{A} / \mathrm{cm}^{2}$ (left and close to SNIC) in phase plane are shown Fig. A.1(b). 

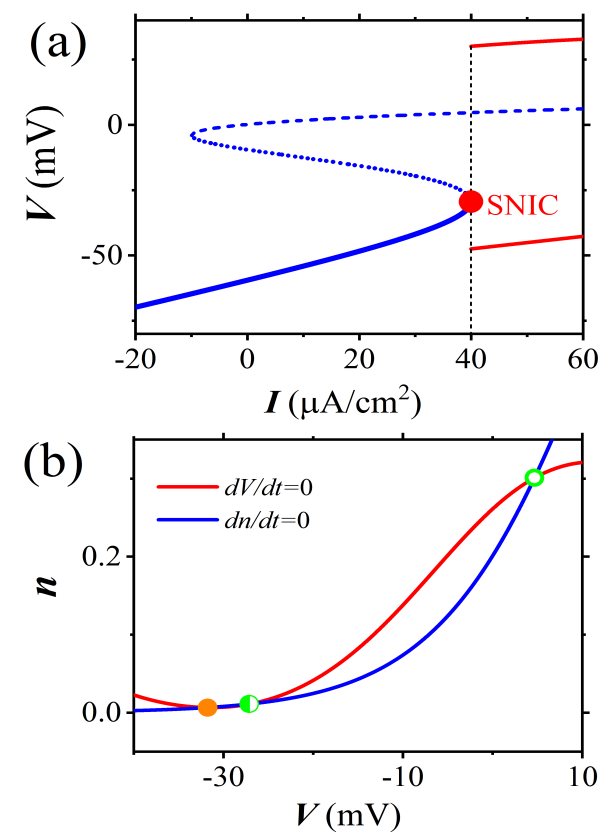

Fig. A.1: The dynamics of the ML model. (a) The bifurcation diagram respect to $I$. The solid blue line is for the stable node, dotted blue for the saddle, and dashed blue for the unstable focus. The red curves denote the extreme values of the stable limit cycle. The symbol SNIC (red point) represents a saddle-node on an invariant circle and occurs at $I_{\mathrm{SNIC}} \approx 39.96 \mu \mathrm{A} / \mathrm{cm}^{2} ;(\mathrm{b})$ The phase portrait at $I=39.95 \mu \mathrm{A} / \mathrm{cm}^{2}$. The intersection points between the nullclines $d V / d t=0$ (red) and $d n / d t=0$ (blue) denote the equilibrium points, i.e., the stable node (orange point), saddle (half-filled point), and unstable focus (hollow point), which are the same as those shown in Fig. 16 in body text. 